\section{Birsel Ayrulu Billur Barshan}

Department of Electrical and Electronics Engineering Bilkent University Bilkent 06533 Ankara, Turkey

\title{
Identification of Target Primitives with Multiple Decision-Making Sonars Using Evidential Reasoning
}

\begin{abstract}
In this study, physical models are used to model reflections from target primitives commonly encountered in a mobile robot's environment. These targets are differentiated by employing a multitransducer pulselecho system that relies on both time-of-flight data and amplitude in the feature-fusion process, allowing more robust differentiation. Target features are generated as being evidentially tied to degrees of belief, which are subsequently fused by employing multiple logical sonars at geographically distinct sites. Feature data from multiple logical sensors are fused with Dempster's rule of combination to improve the performance of classification by reducing perception uncertainty. Using three sensing nodes, improvement in differentiation is between $10 \%$ and $35 \%$ without false decision, at the cost of additional computation. The method is verified by experiments with a real sonar system. The evidential approach employed here helps to overcome the vulnerability of the echo amplitude to noise, and enables the modeling of nonparametric uncertainty in real time.
\end{abstract}

\section{Introduction}

There is no single sensor that perfectly detects, locates, and identifies targets under all circumstances. Although some sensors are more accurate at locating and tracking objects, they may not provide identity information, or vice versa, pointing to the need for combining data from multiple sensors via data-fusion techniques. The primary aim of data fusion is to combine data from multiple sensors to perform inferences that may not be possible from a single sensor. Data-fusion applications span a wide domain, including automatic target recognition, mobile-robot navigation, target tracking, aircraft navigation, and teleoperations (Steinberg 1987; Blackman

The International Journal of Robotics Research,

Vol. 17, No. 6, June 1998, pp. 598-623,

(c) 1998 Sage Publications, Inc. and Broida 1990; Hall 1992; Murphy 1993). In robotics applications, data fusion enables intelligent sensors to be incorporated into the overall operation of robots so that they can interact with and operate in unstructured environments, without the complete control of a human operator (Luo and Lin 1988).

Data fusion can be accomplished by using geometrically, geographically, or physically different sensors at different levels of representation, such as signal-level, pixel-level, feature-level, and symbol-level fusion. In this study, physically identical sonar sensors are employed to combine information when they are located at geographically different sensing sites. Feature-level fusion is used to provide a system performing an object-recognition task with additional features that can be used to increase its recognition capabilities.

One mode of sensing that is potentially useful and cost effective for mobile-robot applications is sonar. Since acoustic sensors are light, robust, and inexpensive devices, they are widely used in applications such as navigation of autonomous vehicles through unstructured environments (Kuc and Siegel 1987; Kuc and Viard 1991), map building (Crowley 1985; Leonard and Durrant-Whyte 1991), target tracking (Kuc 1993), and obstacle avoidance (Borenstein and Koren 1988). Although there are difficulties in the interpretation of sonar data owing to multiple specular reflections, the poor angular resolution of sonar, and the need to establish correspondence between multiple echoes on different receivers (Peremans, Audenaert, and Campenhout 1993; Kleeman and Kuc 1995), these difficulties can be overcome by employing accurate physical models for the reflection of sonar. Sensory information from a single sonar has poor angular resolution and is not sufficient to differentiate the most commonly encountered target primitives (Barshan and Kuc 1990). Therefore, many applications require multiple sonar configurations. The most popular sonar ranging system is based on the time-of-flight (TOF) measurement, which is the 
time elapsed between the transmission of a pulse and its reception. Since the amplitude of sonar signals is prone to environmental conditions and since the standard electronics for the commonly used Polaroid transducers (Polaroid 1990) do not provide the echo amplitude directly, most sonar systems exploit only the TOF information. Differential TOF models of planes, edges, corners, and cylinders have been used by several researchers in map-building, robot-localization, and target tracking applications. In Bozma (1992), using a single mobile sensor for map building, edges are differentiated from planes and corners from a single location. Planes and corners are differentiated by scanning from two separate locations using TOF information from complete sonar scans of the targets. In Leonard (1990) and Peremans et al. (1993), similar approaches have been proposed to identify these targets as beacons for mobile-robot localization. Manyika has applied differential TOF models to target tracking (Manyika and Durrant-Whyte 1994).

For improved target classification, multitransducer pulse/echo systems that rely on both TOF and amplitude information can be employed. In earlier work by Barshan and Kuc, a methodology based on TOF and amplitude information is introduced to differentiate planes and corners using a statistical error model for the noisy signals (Barshan and Kuc 1990). Here, this work is extended to develop algorithms that cover additional target types and fuse the decisions of multiple sensing agents using evidential reasoning. Uncertain environmental data acquired by multiple sonars at distinct geographical sites is used for target recognition. First, the ultrasonic reflection process from commonly encountered target primitives is modeled such that sonar pairs become evidential logical sensors. Logical sensors, as opposed to physical sensors that simply acquire real data, process real sensory data to generate perception units that are context-dependent interpretations of the real data (Nazlibilek and Erkmen 1993). By processing the real data, logical sensors classify the target primitives. An automated perception system for mobile robots fusing uncertain sensory information must be reliable in the sense that it is predictable. Therefore, quantitative approaches to uncertainty are needed. These considerations favor measure-based methods for handling sensory data (both physical and logical) at different levels of granularity related to the resolution of the data, as well as the time constants of the different sensors. The sensor-integration problem can be abstracted in a conceptual model where uncertainty about evidence and knowledge can be measured and systematically reduced. To overcome the vulnerability of echo amplitude to noise, multiple sonar sensors are used in the decision-making process. Decisions of these sensing agents are then integrated using Dempster's rule of combination.

Section 2 explains the sensing configuration used in this study, and introduces the target primitives. A differentiation algorithm that is employed to identify the target primitives is also provided in the same section. In Section 3, the belief-

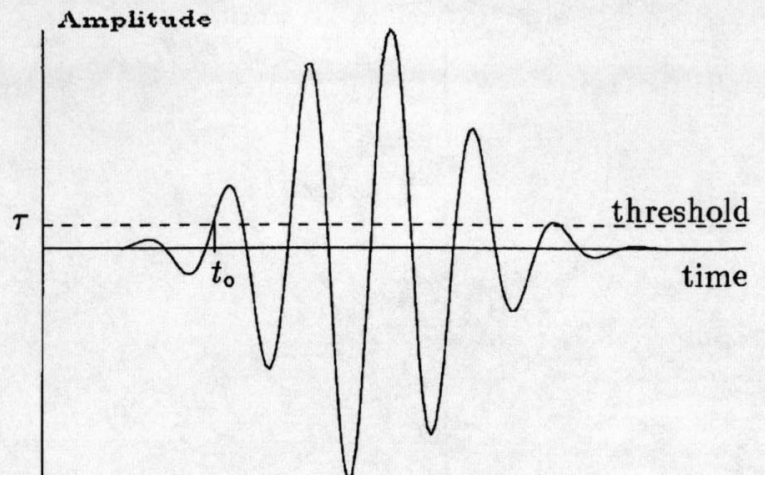

Fig. 1. A typical echo of the ultrasound ranging system.

assignment process is described, which is based on both TOF and amplitude characteristics of the data. Also included is a description of feature and location fusion when multiple sonar-sensing nodes are used. Consensus of multiple sensors at different sites is achieved by using Dempster's rule of combination, and the sensitivity to different levels of amplitude noise is investigated. Simulation results are provided in Section 4. In Section 5, the methodology is verified experimentally by assigning belief values to the TOF and amplitude characteristics of the target primitives, based on real data. Further experiments are conducted in an uncluttered rectangular room where feature and location fusion processes are demonstrated by employing one to three sensing nodes. In the last section, concluding remarks are made and directions for future research are motivated.

\section{Sonar Sensing and Target Differentiation Algorithm}

In this section, basic principles of sonar sensing are reviewed. The sensing configuration and the target primitives that are used in this study are described. A differentiation algorithm is developed to identify and locate the target primitives from the measurements of a single logical sensor.

\subsection{Physical Reflection Models of Sonar from Different Target Primitives}

The most popular sonar ranging system is the TOF system. In this system, an echo is produced when the transmitted pulse encounters an object and a range value $r$ is produced when the echo amplitude waveform first exceeds a preset threshold level $\tau$, as shown in Figure 1:

$$
r=\frac{c t_{\circ}}{2}
$$

Here, $t_{0}$ is the TOF of the echo signal at which the echo amplitude first exceeds the threshold level, and $c$ is the speed 


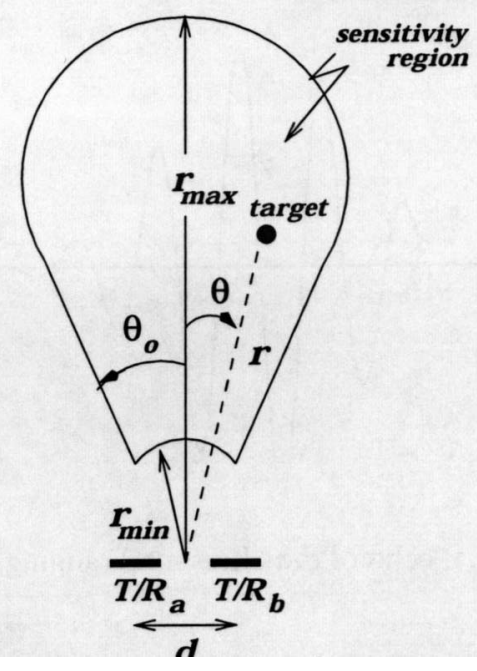

Fig. 2. Sensitivity region of an ultrasonic transducer pair.

of sound in air. ${ }^{1}$ Assuming additive Gaussian-distributed noise, $\tau$ is usually set equal to 4 to 5 times the value of the noise standard deviation, which is estimated based on experimental data.

In this study, the far-field model of a piston-type transducer having a circular aperture is used (Zemanek 1971). The amplitude of the echo decreases with inclination angle $\theta$, which is the deviation angle of the target from normal incidence, as illustrated in Figure 2. The echo amplitude falls below the threshold level when $|\theta|>\theta_{0}$, where $\theta_{0}$ is the beam angle that depends on the aperture size and the resonant frequency of the transducer as

$$
\theta_{\circ}=\sin ^{-1}\left(\frac{0.61 c}{a f_{\circ}}\right)
$$

Here, $a$ is the transducer aperture radius, and $f_{\circ}$ is the resonant frequency of the transducer (Zemanek 1971).

With a single transducer, it is not possible to estimate the azimuth of a target with better resolution than the angular resolution of sonar, which is approximately $2 \theta_{0}$. In the present system, two identical acoustic transducers $a$ and $b$ with center-to-center separation $d$ are employed to improve the angular resolution, as illustrated in Figure 2. Each transducer can operate both as transmitter and receiver by construction. The typical shape of the sensitivity region of an ultrasonic transducer pair is shown in Figure 2. The extent of this region is in general different for each target type, since geometrically or physically different targets exhibit different reflection properties. The word target is used here to refer to any environmental feature that is capable of being observed by a sonar sensor.

1. $c=331.4 \sqrt{\frac{T}{273}} \mathrm{~m} / \mathrm{s}$, where $T$ is absolute temperature in Kelvin. At room temperature $(T=293 \mathrm{~K}), c=343.3 \mathrm{~m} / \mathrm{s}$.
In this study, the target primitives modeled are plane, corner, acute corner, edge, and cylinder, whose horizontal cross sections are illustrated in Figure 3. These target primitives constitute the basic building blocks for most of the surfaces likely to exist in an uncluttered robot environment. Since the wavelength of sonar $(\lambda \cong 8.6 \mathrm{~mm}$ at $40.0 \mathrm{kHz}$ ) is much larger than the typical roughness of object surfaces encountered in laboratory environments, targets in these environments reflect acoustic beams specularly, like a mirror (Morse and Ingard 1968). Hence, while modeling the received signals from these targets, all reflections are considered to be specular. This allows transducers both transmitting and receiving to be viewed as a separate transmitter $T$ and virtual receiver $R$ in all cases (Kuc and Siegel 1987).

Detailed physical reflection models of these target primitives with corresponding echo-signal models are provided in the Appendix.

\subsection{Target Differentiation Algorithm}

In the differentiation of the target primitives discussed in this section, both TOF and amplitude characteristics are used. In Figures 4 and 5, TOF characteristics of various target primitives are given over the range $\theta \in\left[-60^{\circ}, 60^{\circ}\right]$ for a wide-beam transducer. The TOF characteristics of plane, corner, edge, and cylinder have almost the same form as illustrated in Figure 4. However, Figure 5 indicates that the TOF characteristics of the acute corner are significantly different than those of other targets. Let $t_{a b}(\theta)$ denote the TOF reading extracted at angle $\theta$ from $A_{a b}(r, \theta, d, t)$, which is the signal transmitted by $a$ and received by $b$, modeled in the Appendix. The difference in the TOF characteristics of the acute corner is exploited by the following algorithm to differentiate the acute corner from the other targets.

\section{Acute corner differentiation algorithm}

- If $\left[t_{a a}(\theta)-t_{a b}(\theta)\right]>k_{t} \sigma_{t}$ and $\left[t_{b b}(\theta)-t_{a b}(\theta)\right]>k_{t} \sigma_{t}$ then acute corner;

$$
\begin{aligned}
& \text { - else if }\left[t_{a b}(\theta)-t_{a a}(\theta)\right]>k_{t} \sigma_{t} \text { or }\left[t_{a b}(\theta)-t_{b b}(\theta)\right]> \\
& k_{t} \sigma_{t} \\
& \text { then plane, corner, edge, or cylinder. }
\end{aligned}
$$

In this algorithm, $\sigma_{t}$ is the standard deviation of the TOF estimate, which is in general nonlinearly related to the additive noise on the signal amplitude. This relationship is investigated in (Ayrulu 1996). A multiple of $\sigma_{t}, k_{t} \sigma_{t}$, is used to improve the robustness of the differentiation algorithm to noise (Ayrulu 1996).

Note that if a new decision on the target type is to be made at each value of $\theta$ as proposed in the algorithm, an acute corner and a corner cannot be differentiated over $a \pm 1^{\circ}$ interval around $\theta=0^{\circ}$. This is because the qualitative TOF 


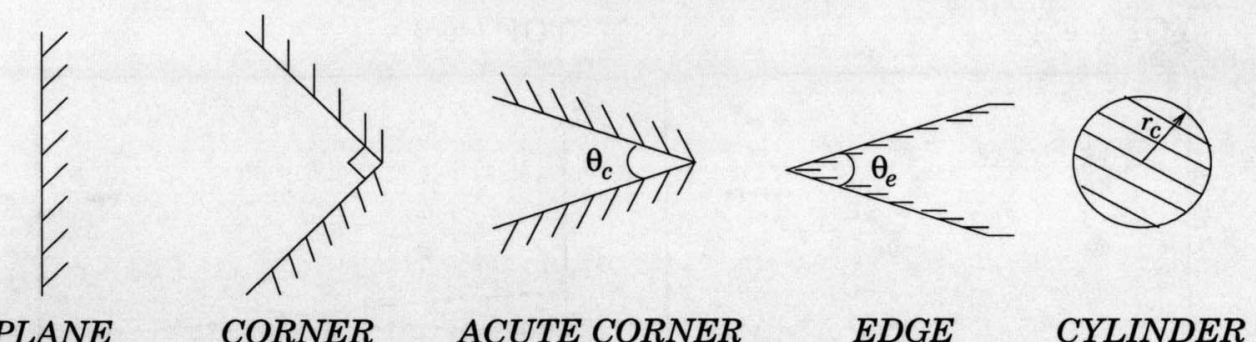

Fig. 3. Target primitives modeled and differentiated in this study.
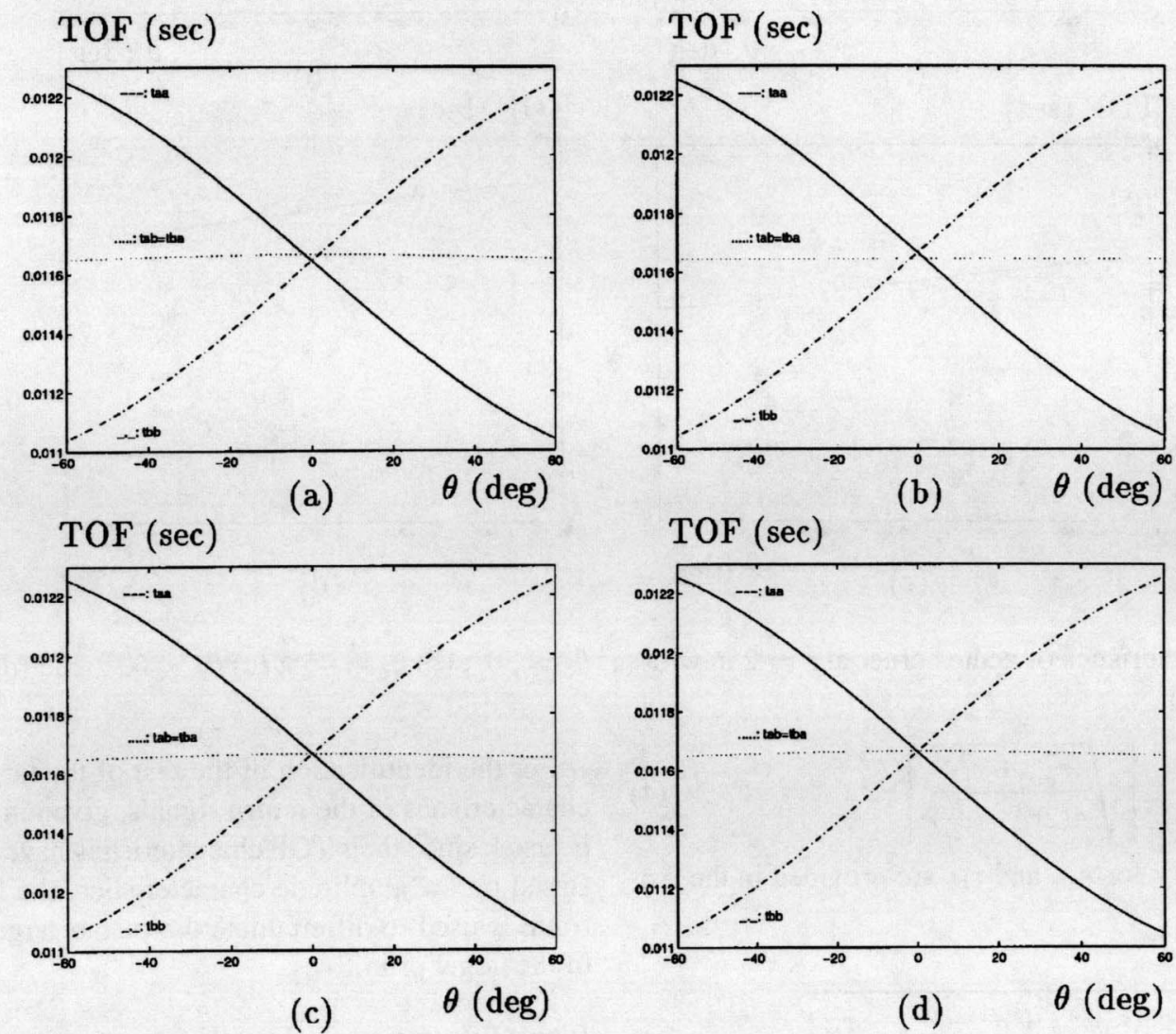

Fig. 4. The TOF characteristics of targets when the target is at $r=2 \mathrm{~m}$ : (a) plane; (b) corner; (c) edge with $\theta_{e}=90^{\circ}$; and (d) cylinder with $r_{c}=20 \mathrm{~cm}$.

characteristics of a corner are the same as that of an acute corner in this interval, as illustrated in Figures $4 \mathrm{~b}$ and 5. However, after mistakenly identifying a corner as an acute corner, the wedge angle of the acute corner will be computed as $90^{\circ}$ in this small interval, as verified experimentally in Section 5 . Hence, if the differentiation algorithm initially detects an acute corner but calculates the wedge angle to be around $90^{\circ}$, the final decision will be a corner. For $\theta$ values outside the interval $\left[-20^{\circ}, 20^{\circ}\right]$, an acute corner of $\theta_{c}=60^{\circ}$ cannot be differentiated from the other target primitives, since its TOF characteristics resemble those of other target primitives for these $\theta$ values. Similarly, acute corners of $\theta_{c}=45^{\circ}$ and $\theta_{c}=30^{\circ}$ cannot be differentiated when $\theta$ is outside the intervals $\left[-45^{\circ}, 45^{\circ}\right]$ and $\left[-55^{\circ}, 55^{\circ}\right]$, respectively. Therefore, acute corners of wedge angle less than $60^{\circ}$ can be reliably differentiated from the rest of the target primitives when $\theta \in\left[-20^{\circ}, 20^{\circ}\right]$. If $\theta_{c}>60^{\circ}$, the differentiation is not reliable, since the TOF characteristics are very similar to those of other targets.

The azimuth $\theta$ and angle $\theta_{c}$ of the acute corner can be estimated as

$$
\theta=\sin ^{-1}\left[\frac{\left(r_{b b}^{2}-r_{a a}^{2}\right)\left(2 r^{2}+\frac{d^{2}}{2}\right)}{2 d r\left(r_{b b}^{2}+r_{a a}^{2}\right)}\right], \text { and }
$$




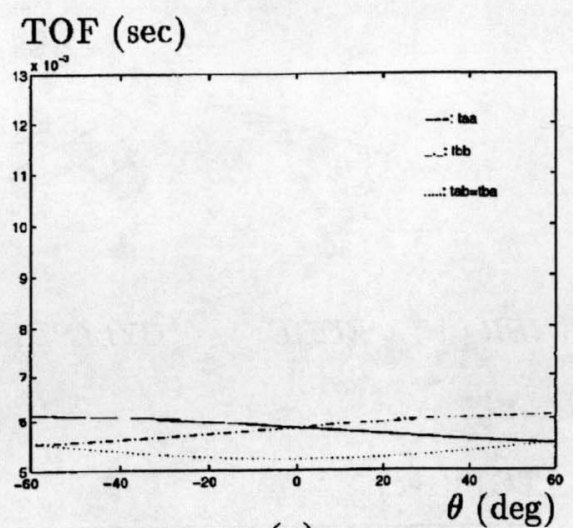

(a)

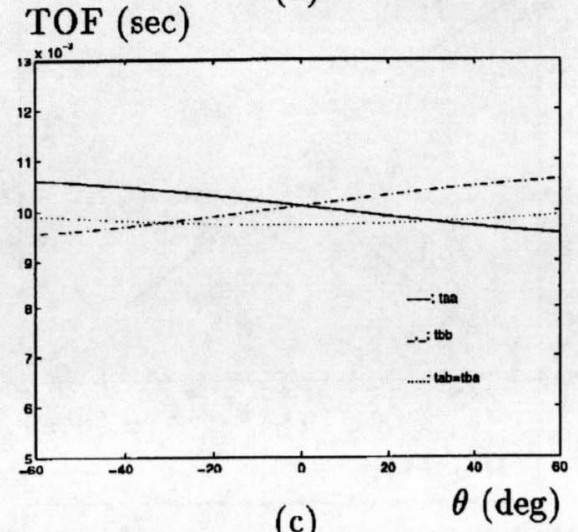

(c)

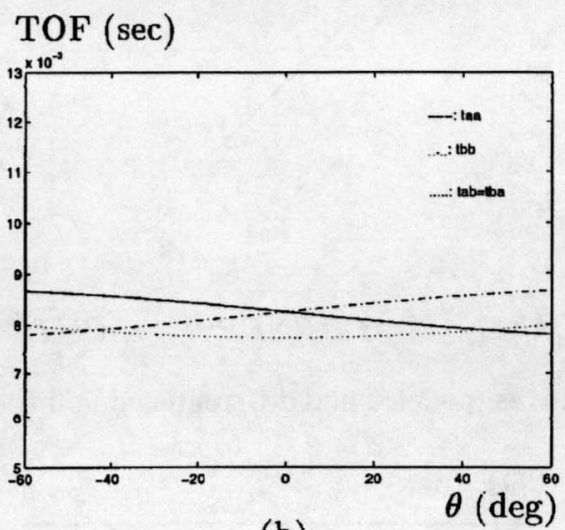

(b)

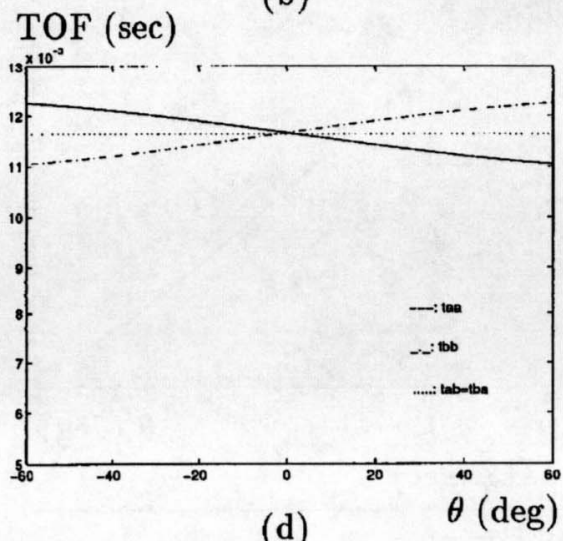

(d)

Fig. 5. TOF characteristics of acute corner at $r=2 \mathrm{~m}$ with (a) $\theta_{c}=30^{\circ}$; (b) $\theta_{c}=45^{\circ}$; (c) $\theta_{c}=60^{\circ}$; and (d) $\theta_{c}=90^{\circ}$.

$$
\theta_{c}=\sin ^{-1}\left[\sqrt{\frac{r_{a a}^{2}+r_{b b}^{2}}{4\left(2 r^{2}+\frac{d^{2}}{2}\right)}}\right],
$$

where the geometry for $r_{a a}$ and $r_{b b}$ are provided in the Appendix.

For $\theta=0^{\circ}$,

$$
r=\sqrt{\frac{d^{2}\left(r_{a b} r_{b b}+d^{2}-r_{a b}^{2}-r_{b b}^{2}\right)}{4\left(r_{a b}-r_{b b}\right)^{2}-d^{2}}} .
$$

To estimate the range $r$ for $\theta \neq 0^{\circ}$, a second-order polynomial equation must be solved:

$$
A x^{2}+B x+C=0 \quad \text { where } \quad x=2 r^{2}+\frac{d^{2}}{2} .
$$

The coefficients of this polynomial are:

$$
\begin{aligned}
A & =\left(\frac{r_{a a}^{2}-r_{b b}^{2}}{r_{b b}^{2}}\right)^{2} \\
B & =\left(\frac{r_{a a}^{2}+r_{b b}^{2}}{r_{b b}^{2}}\right)\left\{r_{a a}^{2}-\frac{1}{r_{b b}^{2}}\left[\left(r_{a a}^{2}+r_{b b}^{2}\right)\left(r_{a b}^{2}+d^{2}\right)\right.\right. \\
& \left.\left.-\left(r_{a b}^{2}-d^{2}\right)^{2}\right]\right\} \\
C & =d^{2} r_{a b}^{2}\left(\frac{r_{a a}^{2}+r_{b b}^{2}}{r_{b b}^{2}}\right)^{2}
\end{aligned}
$$

For the identification of the rest of the targets, amplitude characteristics of the return signals, given in Figure 6, must be used, since their TOF characteristics have the same form. Based on the amplitude characteristics, the following algorithm is used to differentiate the planar target from the rest of the target primitives.

\section{Plane differentiation algorithm}

- If $\left[A_{a a}(\theta)-A_{a b}(\theta)\right]>k_{A} \sigma_{A}$ and $\left[A_{b b}(\theta)-A_{a b}(\theta)\right]>$ $k_{A} \sigma_{A}$

then plane with

$$
\begin{aligned}
& r=\frac{r_{a}+r_{b}}{2} \\
& \theta=\sin ^{-1}\left(\frac{r_{b}-r_{a}}{d}\right) ;
\end{aligned}
$$

- else if $\left[A_{a b}(\theta)-A_{a a}(\theta)\right]>k_{A} \sigma_{A}$ or $\left[A_{a b}(\theta)-\right.$ $\left.A_{b b}(\theta)\right]>k_{A} \sigma_{A}$

then corner, edge, or cylinder.

Here, $A_{a a}(\theta), A_{a b}(\theta)$, and $A_{b b}(\theta)$, respectively, denote the maximum values of $A_{a a}(r, \theta, d, t), A_{a b}(r, \theta, d, t)$, and 


\section{Amplitude}

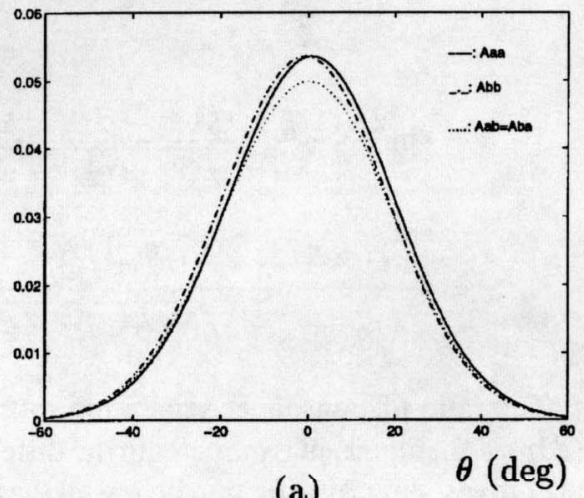

(a)

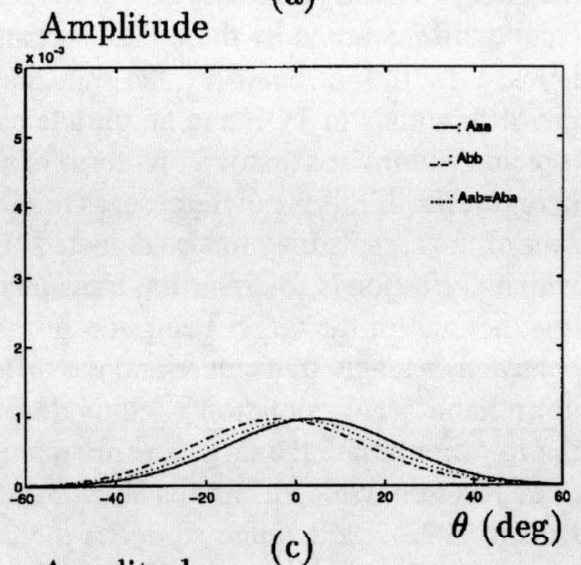

(c)

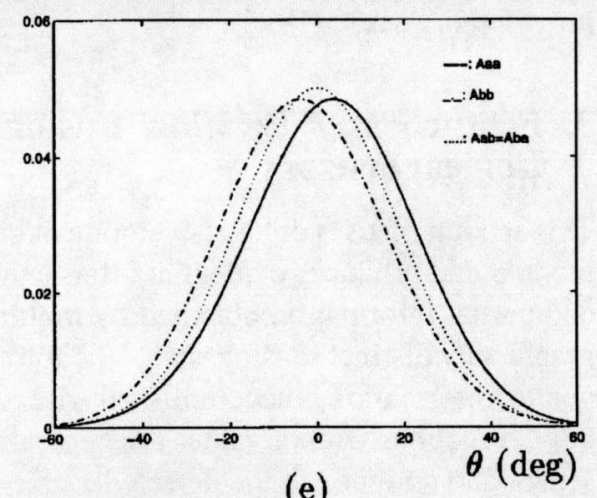

\section{Amplitude}

(e)
Amplitude

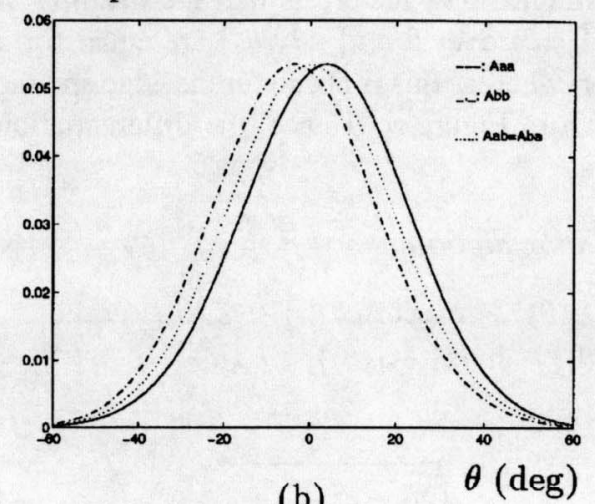

(b)

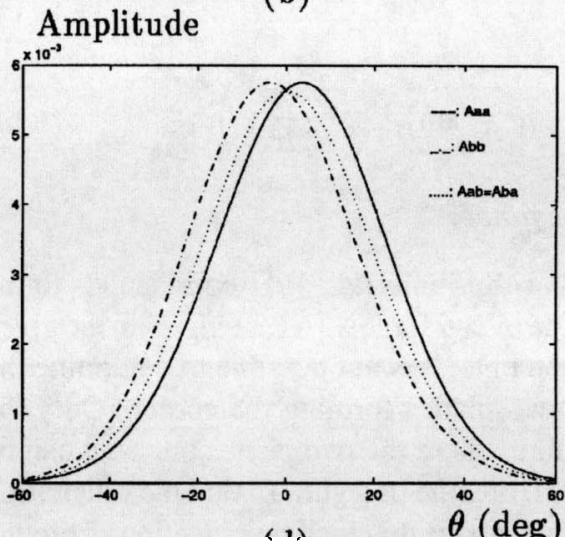

Fig. 6. Amplitude characteristics at $r=2 \mathrm{~m}$ when the target is a (a) plane; (b) corner; (c) edge with $\theta_{e}=90^{\circ}$; (d) cylinder with $r_{c}=20 \mathrm{~cm}$; (e) acute corner with $\theta_{c}=60^{\circ}$. 
$A_{b b}(r, \theta, d, t)$ over time at angle $\theta$. Functional forms of the latter are provided in the Appendix. The $r_{a}$ and $r_{b}$ are the perpendicular distances of the respective transducers from the target, whose geometries are also included in the Appendix.

To differentiate a corner target from an edge or cylinder, amplitude characteristics over the range $\theta \in\left[-\theta_{0}, \theta_{0}\right]$ are studied. The distinguishing feature is that the maxima of $A_{a a}(\theta), A_{a b}(\theta), A_{b b}(\theta)$ over $\theta \in\left[-\theta_{\circ}, \theta_{\circ}\right]$ are equal for a right-angle corner, whereas this is not so for the edge and the cylinder, as shown in Figure 6. Hence, the differentiation algorithm follows.

\section{Corner differentiation algorithm}

- If $\left[\max \left\{A_{a a}(\theta)\right\}-\max \left\{A_{b b}(\theta)\right\}<k_{A} \sigma_{A}\right]$ and $\left[\max \left\{A_{b b}(\theta)\right\}-\max \left\{A_{a b}(\theta)\right\}<k_{A} \sigma_{A}\right]$

then corner with

$$
\begin{aligned}
& r=\sqrt{\frac{r_{a}^{2}+r_{b}^{2}-\frac{d^{2}}{2}}{2}} \\
& \theta=\sin ^{-1}\left(\frac{r_{b}^{2}-r_{a}^{2}}{2 d r}\right) ;
\end{aligned}
$$

else edge or cylinder.

In the above algorithm, $\max \left\{A_{a a}(\theta)\right\}$ corresponds to the maximum amplitude over $\theta$ for $\theta \in\left[-\theta_{0}, \theta_{\mathrm{o}}\right]$. With the given number of measurements, it is not possible to determine the orientation of the two planes forming the corner. Only the orientation of the line where the two planes intersect can be found with respect to the line of sight. To find the orientation of the planes, measurements that include reflections from the two constituent planes are necessary.

In the above algorithms, noise multiplicity factors $k_{A}$ and $k_{t}$ provide robustness for the differentiation process. Simulation results for integer values of $k_{A}$ and $k_{t}$ between 0 and 6 are provided in Ayrulu (1996), which indicate that for the desired level of robustness, it is appropriate to set these equal to one. In situations where a greater level of robustness is desired, larger values may be employed.

Referring to Figure 6, edge and cylinder targets can be distinguished over a small interval near $\theta=0^{\circ}$. At $\theta=0^{\circ}$, $A_{a a}(0)=A_{a b}(0)=A_{b b}(0)$ for an edge, but this equality is not true for a cylinder. Depending on the radius of the cylinder, it may be possible to differentiate edge and cylinder with this configuration of transducers. An edge is a target with zero radius of curvature. For an edge, expressions for range $r$ and azimuth $\theta$ given in eqs. (12) and (13) are the same as in the case of a corner. In the case of a cylinder, in addition to range and azimuth, the radius of the cylinder can be estimated. The radius of curvature has two limits of interest: As $r_{c} \rightarrow 0$, the characteristics of the cylinder approach those of an edge. On the other hand, as $r_{c} \rightarrow$ $\infty$, the characteristics are more similar to those of a plane.
By assuming the target is a cylinder first and estimating its radius of curvature (Barshan 1996), it may be possible to distinguish these two targets for relatively large values of $r_{c}$. Approximate expressions for the $r, \theta$ and $r_{c}$ estimates are given by

$$
\begin{aligned}
& r \cong \frac{\sqrt{\left(r_{1}+r_{2}\right)^{2}-d^{2}}}{2} \\
& \theta \cong \sin ^{-1}\left[\frac{\left(r_{a}^{2}-r_{b}^{2}\right)+2 r_{c}\left(r_{a}-r_{b}-d\right)}{d \sqrt{\left(r_{1}+r_{2}\right)^{2}-d^{2}}}\right], \text { and } \\
& r_{c} \cong \frac{\frac{\left(r_{1}+r_{2}\right)^{2}}{2}-\left(r_{a}^{2}+r_{b}^{2}\right)}{2\left(r_{b}+r_{a}-\sqrt{\left(r_{1}+r_{2}\right)^{2}-d^{2}}\right)}
\end{aligned}
$$

The ratio of transducer separation to the operating range $(d / r)$ is an important parameter in the differentiation of target primitives, directly affecting how well these target primitives can be differentiated by their TOF and amplitude characteristics. The further apart are the transducers, the larger are the differentials in TOF and amplitude as long as the target remains within the sensitivity patterns of both transducers, as in Figure 7a. If this is not the case, as in Figure 7b, some or all four of the signals may not be detected. In the limit $\frac{d}{r} \rightarrow 0$, which corresponds to either the transducers being too close together and/or the target being too far, the two transducers behave as a single transducer and the differential signals are not reliable. This situation is equivalent to the case of trying to differentiate the targets using a single transducer at a fixed location, which is not feasible (Barshan and Kuc 1990; Bozma 1992). A detailed study on the effect of transducer separation $d$ and range $r$ on the maximum differentials is provided in Ayrulu (1996).

\section{Feature and Position Fusion by Multiple Logical Sensors}

This section focuses on the development of a logical sensing module that produces evidential information from uncertain and partial information obtained by multiple sonars at geographically distinct sensing sites. The formation of such evidential information is accomplished with reasoning based on belief functions. Belief values are generated by each logical sensor and assigned to the detected features. These features and their evidential metric obtained from multiple sonars are then fused using Dempster's rule of combination.

A belief function is a mapping from a class of sets to the interval $[0,1]$ that assigns numerical degrees of support based on evidence (Shafer 1976). This is a generalization of probabilistic approaches, since one is allowed to model ignorance about a given situation. Unlike probability theory, a belief function brings a metric to the intuitive idea that a portion of one's belief can be committed to a set but need not be also 


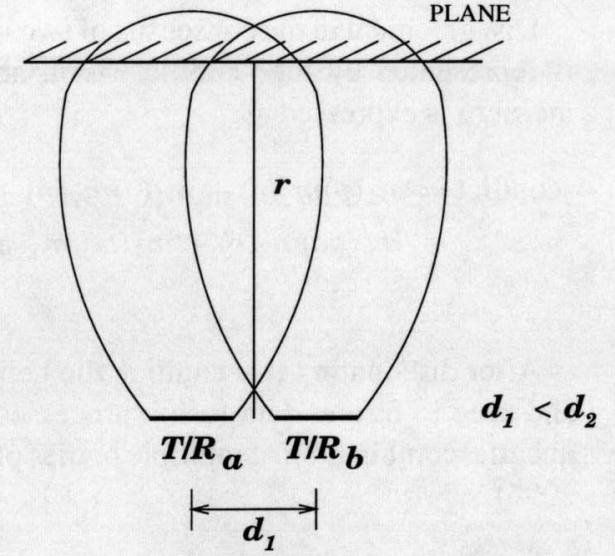

(a)

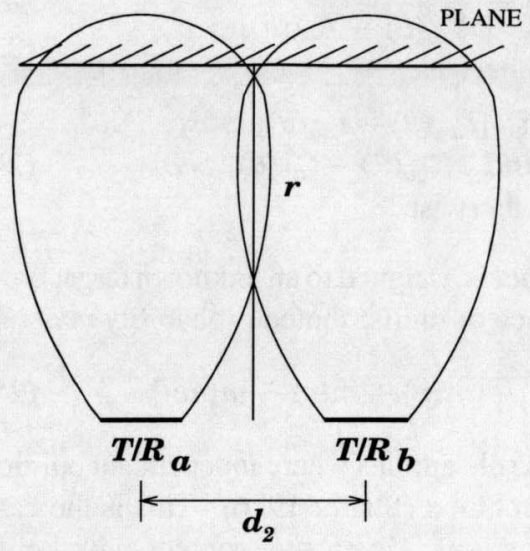

(b)

Fig. 7. A planar target falls (a) within the intersection of the sensitivity patterns of both transducers and (b) outside the intersection of the sensitivity patterns so that cross-signals are not detected.

committed to its complement. In the target classification problem, ignorance corresponds to not having any information on the type of target that the transducer pair is scanning. Dempster-Shafer theory differs from the Bayesian approach by allowing support for more than one proposition at a time, allowing lack of data (ignorance) to be represented. With this approach, full description of conditional (or prior) probabilities are no longer required, and incremental evidence can be easily incorporated. Several researchers have recently started using evidential reasoning in applications such as landmarkbased navigation (Murphy 1996) and map building (Pagac, Nebot, and Durrant-Whyte 1996).

To differentiate the target primitives, differences in the reflection characteristics of these targets are exploited and formulated in terms of basic probability masses. This logical sensor model of sonar perception is novel in the sense that it models the uncertainties associated with the target type, its range, and its azimuth, as detected by each sensor pair. The uncertainty in the measurements of each sensor node is represented by a belief function having target type (or feature) and target location $r$ and $\theta$ as focal elements, with basic probability masses $m($.$) associated with them:$

$$
B F=\{\text { feature, } r, \theta ; m(\text { feature }), m(r), m(\theta)\} .
$$

\subsection{Feature Fusion from Multiple Sonars}

The focus of this section is feature fusion; fusion of targetlocation estimates will be handled in the next section. Logical sensing of the target primitives is accomplished through a metric as degrees of belief assigned to the target primitives, according to the TOF and amplitude characteristics of the received signals described in Section 2. According to the method used in this study, a new decision on the target type is made on-line at each discrete value of $\theta$, based on the differentiation algorithm. Since complete amplitude sonar scans that cover the whole range of $\theta \in\left[-\theta_{0}, \theta_{0}\right]$ must be interpreted to differentiate edge and cylinder from corner, it is possible to differentiate only plane, corner, and acute corner with on-line data processing. However, once complete TOF and amplitude characteristics are obtained for all values of $\theta$, all five targets can be differentiated. Based on TOF and amplitude characteristics of the received signals from plane, corner, and acute corner, basic probability assignment to each feature is made as follows:

$$
m(p)=\left(1-I_{4}\right) I_{1} \frac{\left[A_{a a}(\theta)-A_{a b}(\theta)\right]+\left[A_{b b}(\theta)-A_{a b}(\theta)\right]}{\max \left[A_{a a}(\theta)-A_{a b}(\theta)\right]+\max \left[A_{b b}(\theta)-A_{a b}(\theta)\right]},
$$

$$
m(c)=\left\{\begin{array}{cl}
\left(1-I_{4}\right) \frac{\left(\begin{array}{c}
I_{2}\left[A_{a b}(\theta)-A_{a a}(\theta)\right] \\
+I_{3}\left[A_{a b}(\theta)-A_{b b}(\theta)\right]
\end{array}\right)}{\left(\begin{array}{c}
I_{2} \max \left[A_{a b}(\theta)-A_{a a}(\theta)\right] \\
+I_{3} \max \left[A_{a b}(\theta)-A_{b b}(\theta)\right]
\end{array}\right)} & \text { if } I_{2} \neq 0 \\
0 & \text { or } I_{3} \neq 0
\end{array}\right.
$$

$m(a c)=I_{4} \frac{\left[t_{a a}(\theta)-t_{a b}(\theta)\right]+\left[t_{b b}(\theta)-t_{a b}(\theta)\right]}{\max \left[t_{a a}(\theta)-t_{a b}(\theta)\right]+\max \left[t_{b b}(\theta)-t_{a b}(\theta)\right]}$,

where $A_{a b}(\theta)$ denotes the maximum value of $A_{a b}(r, \theta, d, t)$ (the signal transmitted by $a$ and received by $b$ ), and $t_{a b}(\theta)$ denotes TOF extracted from $A_{a b}(r, \theta, d, t)$ at inclination angle $\theta$ by thresholding. The definitions of $A_{a a}(\theta), A_{b b}(\theta), t_{a a}(\theta)$, and $t_{b b}(\theta)$ are similar. $I_{1}, I_{2}, I_{3}$, and $I_{4}$ are the indicators of the conditions given below:

$$
\begin{aligned}
& I_{1}=\left\{\begin{array}{l}
1 \text { if }\left[A_{a a}(\theta)-A_{a b}(\theta)\right]>\sigma_{A} \\
\text { and }\left[A_{b b}(\theta)-A_{a b}(\theta)\right]>\sigma_{A} \\
0 \text { otherwise, }
\end{array}\right. \\
& I_{2}= \begin{cases}1 & \text { if }\left[A_{a b}(\theta)-A_{a a}(\theta)\right]>\sigma_{A} \\
0 & \text { otherwise, }\end{cases}
\end{aligned}
$$




$$
\begin{gathered}
I_{3}= \begin{cases}1 & \text { if }\left[A_{a b}(\theta)-A_{b b}(\theta)\right]>\sigma_{A} \\
0 & \text { otherwise, }\end{cases} \\
I_{4}= \begin{cases}1 & \text { if }\left[t_{a a}(\theta)-t_{a b}(\theta)\right]>\sigma_{t} \\
\text { and }\left[t_{b b}(\theta)-t_{a b}(\theta)\right]>\sigma_{t} \\
0 & \text { otherwise. }\end{cases}
\end{gathered}
$$

The remaining belief is assigned to an unknown target type, representing ignorance or undistributed probability mass, as

$$
m(u)=1-[m(p)+m(c)+m(a c)] .
$$

Dempster's fusion rule applies where independent opinion sources are to be combined (Shafer 1976). This is the case in the present application. Given two sources with belief functions

$$
\begin{aligned}
B F_{1} & =\left\{f_{i}, m_{1}\left(f_{i}\right)\right\}_{i=1}^{4} \\
& =\left\{p, c, a c, u ; m_{1}(p), m_{1}(c), m_{1}(a c), m_{1}(u)\right\},
\end{aligned}
$$

and

$$
\begin{aligned}
B F_{2} & =\left\{g_{j}, m_{2}\left(g_{j}\right)\right\}_{j=1}^{4} \\
& =\left\{p, c, a c, u ; m_{2}(p), m_{2}(c), m_{2}(a c), m_{2}(u)\right\},
\end{aligned}
$$

consensus is obtained as the orthogonal sum

$$
\begin{aligned}
B F & =B F_{1} \oplus B F_{2}=\left\{h_{k}, m_{c}\left(h_{k}\right)\right\}_{k=1}^{4} \\
& =\left\{p, c, a c, u ; m_{c}(p), m_{c}(c), m_{c}(a c), m_{c}(u)\right\}
\end{aligned}
$$

which is both associative and commutative, with the resulting operation being shown in Table 1. The sequential combination of multiple bodies of evidence can be obtained for $n$ sensor pairs as

$$
B F=\left(\left(\left(B F_{1} \oplus B F_{2}\right) \oplus B F_{3}\right) \ldots \oplus B F_{n}\right) .
$$

Using Dempster's rule of combination,

$$
m_{c}\left(h_{k}\right)=\frac{\sum \sum_{h_{k}=f_{i} \cap g_{j}} m_{1}\left(f_{i}\right) m_{2}\left(g_{j}\right)}{1-\sum \sum_{h_{k}=f_{i} \cap g_{j}=0} m_{1}\left(f_{i}\right) m_{2}\left(g_{j}\right)},
$$

where $\sum \sum_{h_{k}=f_{i} \cap g_{j}=0} m_{1}\left(f_{i}\right) m_{2}\left(g_{j}\right)$ is a measure of conflict.

The consensus belief function representing the featurefusion process has the metrics

$$
\begin{aligned}
m_{c}(p) & =\frac{m_{1}(p) m_{2}(p)+m_{1}(p) m_{2}(u)}{+m_{1}(u) m_{2}(p)} \\
1-\text { conflict } & m_{1}(c) m_{2}(c)+m_{1}(c) m_{2}(u) \\
m_{c}(c) & =\frac{+m_{1}(u) m_{2}(c)}{1-\text { conflict }}, \\
m_{c}(a c) & =\frac{m_{1}(a c) m_{2}(a c)+m_{1}(a c) m_{2}(u)}{1-\text { conflict }}, \text { and } \\
m_{c}(u) & =\frac{m_{1}(u) m_{2}(u)}{1-\text { conflict }} .
\end{aligned}
$$

Disagreement in the consensus of two logical sensing units is represented by the "conflict" term above. The conflict measure is expressed as

$$
\begin{aligned}
\text { conflict } & =m_{1}(p) m_{2}(c)+m_{1}(c) m_{2}(p)+m_{1}(c) m_{2}(a c) \\
& +m_{1}(a c) m_{2}(c)+m_{1}(a c) m_{2}(p)+m_{1}(p) m_{2}(a c) .
\end{aligned}
$$

After discounting this conflict, the beliefs can be rescaled and used in further data-fusion processes, such as in the sequential combination of multiple bodies of evidence (Murphy 1996).

\subsection{Fusion of Range and Azimuth Estimates}

Assignment of belief to range and angle measurements is based on the simple observation that the closer the target is to the face of the transducer, the more accurate is the range reading, and the closer the target is to the line of sight of the transducer, the more accurate is the angle estimate (Barshan 1991). This is due to the physical properties of sonar: signal amplitude decreases with $r$ and with $|\theta|$. At large ranges and larger angular deviations, the signal-to-noise ratio is smaller. The most accurate measurements are obtained along the line of sight $\left(\theta=0^{\circ}\right)$ and at nearby ranges to the sensor pair. Therefore, belief assignments to range and azimuth estimates derived from the TOF measurements are made as follows:

$$
\begin{aligned}
& m(r)=\frac{r_{\max }-r}{r_{\max }-r_{\min }}, \quad \text { and } \\
& m(\theta)=\frac{\theta_{\circ}-|\theta|}{\theta_{\circ}} .
\end{aligned}
$$

Note that, belief of $r$ takes its maximum value of one when $r=r_{\min }$ and its minimum value of zero when $r=r_{\max }$. Similarly, belief of $\theta$ is one when $\theta=0^{\circ}$ and zero when $\theta= \pm \theta_{0}$.

Since each sensor pair takes measurements in its own sensor-centric coordinate frame, the beliefs of range and azimuth information need to be first projected onto a common coordinate system where they can be integrated. This is represented in Figure 8, where erroneous estimates are assumed for $r$ and $\theta$. Then the metric of the fusion process is computed based on these projected values. Due to the noise on the system, estimated range and azimuth values are different than the true values. Suppose $n$ transducer pairs are employed and each pair estimates the range and azimuth of the target as

$$
\left(\hat{r}_{i}, \hat{\theta}_{i}\right) \quad i=1,2, \ldots, n .
$$

The correct position of the target is denoted by

$$
\left(r_{i}, \theta_{i}\right) \quad i=1,2, \ldots, n
$$


Table 1. Target Differentiation by Dempster's Rule of Combination

\begin{tabular}{|c||c|c|c|c|}
\hline$B F_{1} /$ & Plane & Corner & Acute Corner & Unknown \\
$B F_{2}$ & $m_{1}(p)$ & $m_{1}(c)$ & $m_{1}(a c)$ & $m_{1}(u)$ \\
\hline \hline Plane & Plane & 0 & 0 & Plane \\
$m_{2}(p)$ & $m_{1}(p) m_{2}(p)$ & $m_{1}(c) m_{2}(p)$ & $m_{1}(a c) m_{2}(p)$ & $m_{1}(u) m_{2}(p)$ \\
\hline Corner & 0 & Corner & 0 & Corner \\
$m_{2}(c)$ & $m_{1}(p) m_{2}(c)$ & $m_{1}(c) m_{2}(c)$ & $m_{1}(a c) m_{2}(c)$ & $m_{1}(u) m_{2}(c)$ \\
\hline Acute corner & 0 & 0 & Acute corner & Acute corner \\
$m_{2}(a c)$ & $m_{1}(p) m_{2}(a c)$ & $m_{1}(c) m_{2}(a c)$ & $m_{1}(a c) m_{2}(a c)$ & $m_{1}(u) m_{2}(a c)$ \\
\hline Unknown & Plane & Corner & Acute corner & Unknown \\
$m_{2}(u)$ & $m_{1}(p) m_{2}(u)$ & $m_{1}(c) m_{2}(u)$ & $m_{1}(a c) m_{2}(u)$ & $m_{1}(u) m_{2}(u)$ \\
\hline
\end{tabular}

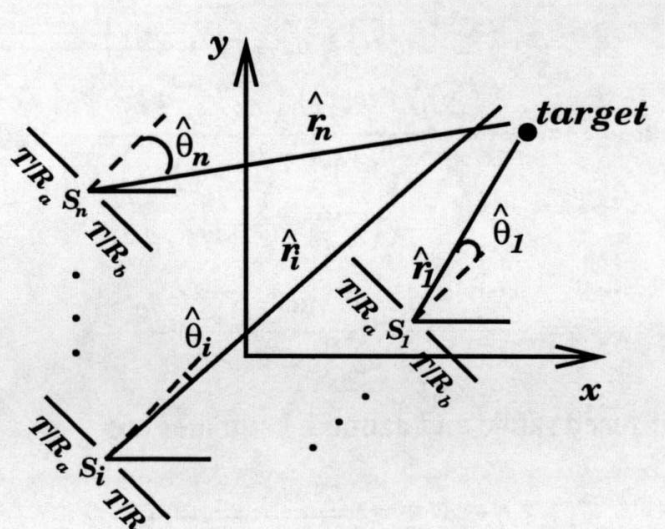

Fig. 8. Common coordinate system for $n$ pairs of sonar sensors.

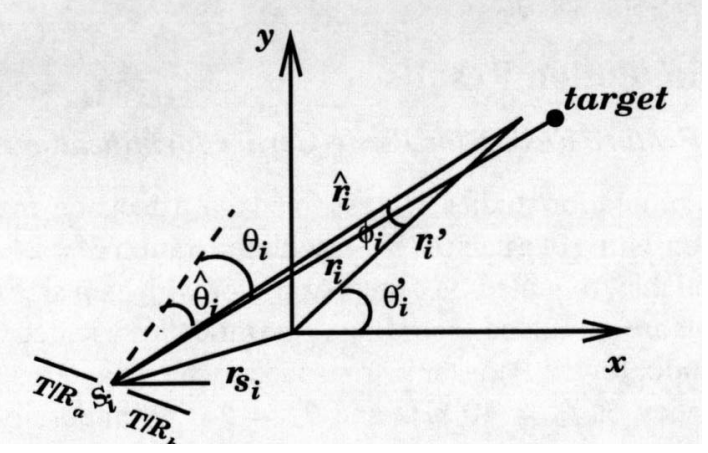

Fig. 9. Projected range and azimuth for transducer pair $i$.

in each sensor's own coordinate frame, while the target is within its sensitivity region. The projected range and azimuth are represented in Figure 9 as

$$
\left(r_{i}^{\prime}, \theta_{i}^{\prime}\right) \quad i=1,2, \ldots, n
$$

Although typically logarithmic relationships are used to relate uncertainty and belief (Pearl 1988), here a simpler linear relationship is chosen to facilitate the analysis:

$$
\frac{\sigma_{p}}{p}=\frac{1-m(p)}{m(p)}
$$

where $p$ corresponds to either the range or azimuth of the target.

Since the range and azimuth estimates are transformed onto a common coordinate frame, uncertainties in the estimated range and azimuth must be represented as uncertainties in the transformed range and azimuth with the transformation below:

$$
\begin{aligned}
\sigma_{r_{i}^{\prime}} & =\sigma_{\hat{r}_{i}}\left|\cos \phi_{i}\right|+\hat{r}_{i} \sigma_{\hat{\theta}_{i}}\left|\sin \phi_{i}\right|, \text { and } \\
r_{i}^{\prime} \sigma_{\theta_{i}^{\prime}} & =\sigma_{\hat{r}_{i}}\left|\sin \phi_{i}\right|+\hat{r}_{i} \sigma_{\hat{\theta}_{i}}\left|\cos \phi_{i}\right|,
\end{aligned}
$$

where $\sigma_{\hat{r}_{i}}$ and $\sigma_{\hat{\theta}_{i}}$ represent uncertainties in the range and azimuth measurements, respectively, and $\phi_{i}$ is the angle between $\hat{r}_{i}$ and $r_{i}^{\prime}$. Since the position of the $i^{\text {th }}$ transducer pair, $r_{s_{i}}$, is known, $\phi_{i}$ can be found from the geometry by using the cosine theorem:

$$
\phi_{i}=\cos ^{-1}\left(\frac{\hat{r}_{i}^{2}+{r_{i}^{\prime}}_{i}^{2}-r_{s_{i}}^{2}}{2 \hat{r}_{i} r_{i}^{\prime}}\right),
$$

where $r_{s_{i}}$ is the distance of the $i^{\text {th }}$ sensor pair from the origin. After projecting the range and azimuth estimates onto a common coordinate system, they are fused into a single range and a single azimuth estimate as follows:

$$
\begin{aligned}
& r_{f}=\frac{\sum_{i=1}^{n} r_{i}^{\prime} m\left(r_{i}^{\prime}\right)}{\sum_{i=1}^{n} m\left(r_{i}^{\prime}\right)}, \text { and } \\
& \theta_{f}=\frac{\sum_{i=1}^{n} \theta_{i}^{\prime} m\left(\theta_{i}^{\prime}\right)}{\sum_{i=1}^{n} m\left(\theta_{i}^{\prime}\right)},
\end{aligned}
$$

where the new belief value in the common coordinate system can be found by solving eq. (41) for $m(p)$.

Beliefs to these combined range and azimuth estimates are assigned by using eqs. (36) and (37). When the system 


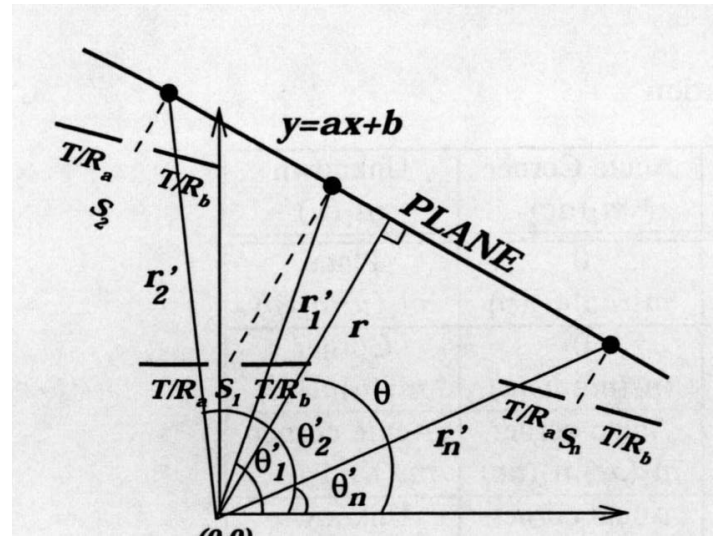

Fig. 10. Position of a plane with respect to each sensor pair.

is noiseless and the location of the target in the common coordinate system is $(r, \theta)$, all estimated range and azimuth values are equal to their true values:

$$
\begin{aligned}
& \hat{r}_{i}=r_{i}, \text { and } \\
& \hat{\theta}_{i}=\theta_{i} .
\end{aligned}
$$

Then the projected and fused range and azimuth estimates are all equal:

$$
\begin{aligned}
r_{i}^{\prime} & =r_{f}=r, \text { and } \\
\theta_{i}^{\prime} & =\theta_{f}=\theta \quad i=1,2, \ldots, n .
\end{aligned}
$$

For the planar target case, which is illustrated in Figure 10, fusion of range and azimuth estimates needs to be modified, because each sensor pair detects the plane at a different position. For this case, a line that represents the plane in 2-D can be estimated using the estimated positions of the plane by all sensor pairs in the common coordinate frame. Then the perpendicular distance between this line and the origin, and the orientation of this line with respect to the origin must be found which yield the fused range and azimuth of this plane.

In 2-D, a planar target can be represented as a line with the equation

$$
y=a x+b .
$$

If range and azimuth measurements from $n$ sensors are available, a weighted least-squares solution (Bar-Shalom 1990) is sought for $a$ and $b$ where the weights and uncertainty are inversely related. The weighted least-squares solution can be found by minimizing the following expression:

$$
\min _{a, b} \sum_{i=1}^{n} w_{i}\left[y_{i}-\left(a x_{i}+b\right)\right]^{2} .
$$

Here,

$$
\begin{aligned}
x_{i} & =r_{i}^{\prime} \cos \theta_{i}^{\prime}, \text { and } \\
y_{i} & =r_{i}^{\prime} \sin \theta_{i}^{\prime} \quad i=1, \ldots, n,
\end{aligned}
$$

and the weights that minimize the mean-square error can be found as (Barshan and Kuc 1990)

$$
w_{i}=\frac{1}{\sigma_{x_{i}}^{2}+\sigma_{y_{i}}^{2}},
$$

where $\sigma_{x_{i}}$ and $\sigma_{y_{i}}$ are found by transforming the uncertainties in $r_{i}^{\prime}$ and $\theta_{i}^{\prime}$ as

$$
\begin{aligned}
& \sigma_{x_{i}}=\sigma_{r_{i}^{\prime}}\left|\cos \theta_{i}^{\prime}\right|+r_{i}^{\prime} \sigma_{\theta_{i}^{\prime}}\left|\sin \theta_{i}^{\prime}\right|, \text { and } \\
& \sigma_{y_{i}}=\sigma_{r_{i}^{\prime}}\left|\sin \theta_{i}^{\prime}\right|+r_{i}^{\prime} \sigma_{\theta_{i}^{\prime}}\left|\cos \theta_{i}^{\prime}\right| .
\end{aligned}
$$

Note that here there is no need to normalize the sum of the weights to one. The weighted least-squares solution is

$$
\begin{gathered}
\left(\sum_{i=1}^{n} w_{i}\right)\left(\sum_{j=1}^{n} w_{j} x_{j} y_{j}\right) \\
a_{\mathrm{WLS}}=\frac{-\left(\sum_{i=1}^{n} w_{i} x_{i}\right)\left(\sum_{j=1}^{n} w_{j} y_{j}\right)}{\left(\sum_{i=1}^{n} w_{i}\right)\left(\sum_{j=1}^{n} w_{j} x_{j}^{2}\right)}, \text { and } \\
-\left(\sum_{i=1}^{n} w_{i} x_{i}\right)^{2} \\
m_{\mathrm{WLS}}=\frac{\sum_{i=1}^{n} w_{i} y_{i}-a_{\mathrm{WLS}} \sum_{i=1}^{n} w_{i} x_{i}}{\sum_{i=1}^{n} w_{i}}
\end{gathered}
$$

and the fused range and azimuth estimates are

$$
\begin{aligned}
& r_{f}=r=\frac{m_{\mathrm{WLS}}}{\sqrt{a_{\mathrm{WLS}}^{2}+1}}, \text { and } \\
& \theta_{f}=\theta=\tan ^{-1}\left(-\frac{1}{a_{\mathrm{WLS}}}\right) .
\end{aligned}
$$

\section{Simulation Results}

\subsection{Feature Fusion for Plane-Corner Identification}

In the simulation studies, it is assumed that a decision-making unit consisting of a pair of sonars with separation $d=24.0 \mathrm{~cm}$ is available, mounted on a stepper motor with step size $0.9^{\circ}$. Signals are simulated according to the models presented in the Appendix for the Panasonic transducer, which has a resonant frequency of $f_{\circ}=40 \mathrm{kHz}$ and $\theta_{\circ}=54^{\circ}$. Temporally and spatially uncorrelated zero-mean additive Gaussian noise of standard deviation $\sigma_{A}$ is added to the echo signals. At each step of the motor, a pulse is transmitted, and four TOF and four amplitude measurements are recorded. The unit scans an uncluttered area which is a $1.4 \mathrm{~m} \times 1.0 \mathrm{~m}$ rectangular room for $\phi \in\left[-180^{\circ}, 180^{\circ}\right]$ in order to detect corners and planar walls.

The results of the belief assignment process for a single transducer pair located at the center of the room are given in Figure 11. In this figure, $m(p)$ clearly indicates that the plane feature is recognized with high beliefs at right angles around $0^{\circ}, \pm 90^{\circ}, \pm 180^{\circ}$, and with highest beliefs in range than corner, since planes lie at closer proximity to the sensor 
*:position of the sensor pair, o: PLANE , +: CORNER
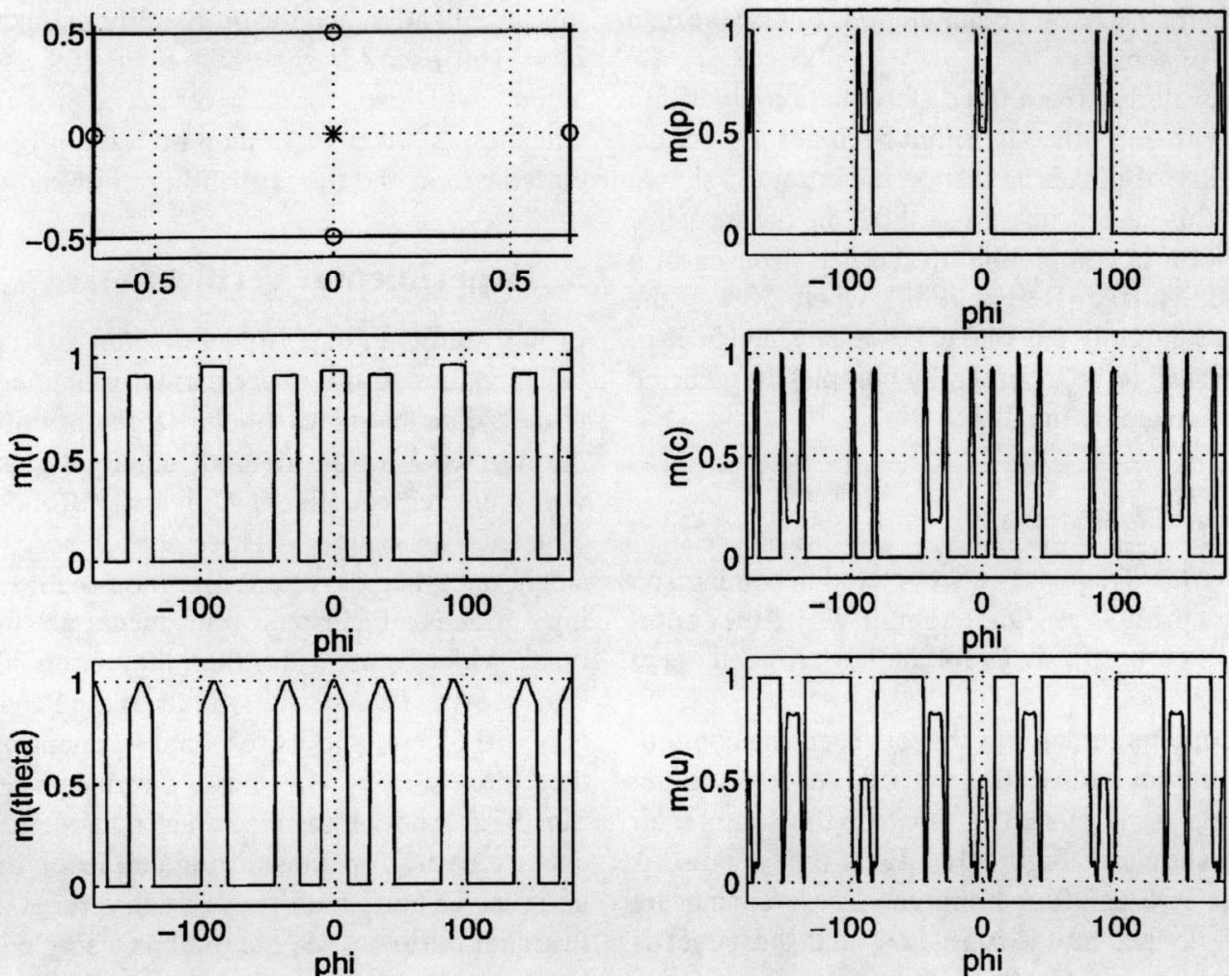

Fig. 11. Belief assignment with information from a single transducer pair.

than corners. For larger inclination angles, these four planes are confused with corners, because the tails of the amplitude characteristics of a plane and corner are similar. The belief $m(c)$ shows that the four corners of the room are identified with highest belief values around $\pm 45^{\circ}$ and $\pm 135^{\circ}$. The belief chop in the middle of each corner belief curve reflects a pin-type rise in uncertainty at these locations. This is due to the amplitude characteristics of the corner. At $+\varepsilon,-\varepsilon$ degrees to the left or to the right of this line, higher beliefs are generated in the recognition of a corner. In the angular interval between the identification of plane and that of corner, there exists a region of high uncertainty in $m(u)$ due to no return signal being available. In this case, neglecting multiple reflections of third and higher orders, all transmitted waveforms bounce off the room boundaries, and no return signal is recorded. Thus, $m(r)=m(\theta)=0$.

Further simulation studies were performed with three identical logical sensors located at different positions in the $1.4 \mathrm{~m} \times 1.0 \mathrm{~m}$ rectangular room. The decisions of the three pairs are combined so as to perform the feature fusion by employing Dempster's rule of combination. The locations of these transducer pairs are $(0.0,0.0),(-0.1,0.1)$, and $(0.1,0.1)$ in meters, where the origin is taken as the center of the room. All transducer pairs are assumed to rotate on stepper motors with step size $0.9^{\circ}$. These units scan the room for $\phi \in\left[-180^{\circ}, 180^{\circ}\right]$. At each step, transducer pairs collect data from the target at the same step angle $\phi$, and the decisions of all pairs at this angle are fused. To calculate probabilities of correct classification, misclassification, and lack of target identification, data is collected for $\phi \in\left[-180^{\circ}, 180^{\circ}\right]$ three times, which corresponds to about 1,200 decisions.

The classification results for each transducer pair and the data fusion using three transducer pairs are given in Figure 12. For a maximum echo-amplitude value of 0.3 , amplitude noise standard deviation of 0.02 corresponds to $50 \%$ of the maximum signal-amplitude differences. For $\sigma_{A} \geq 0.03$, differential signal levels are comparable to the noise level, and it becomes impossible to detect these differences. In Figure $12 \mathrm{~b}-12 \mathrm{~d}$, the probability of misclassification with one pair is almost zero for all the noise standard deviation values, owing to the inclusion of $\sigma_{A}$ in the classification algorithms. The probability of correct classification with the fusion of three pairs can be seen in Figure 12e. The improvement in the probability of correct classification is shown in Figure 12f. Here, the probability of correct classification is derived from the consensus of three logical sonars, illustrating how fusion provides an increase in evidential support that raises the probability of correct classification when compared to that of a single transducer pair. The improvement is between $10 \%$ and $35 \%$ for $\sigma_{A}<0.03$, becoming smaller for larger values of 
$\sigma_{A}$. Of course, this is at the increased cost of time to collect more data and do the necessary computations to fuse the data from three pairs of sensors.

When $\sigma_{A}$ is excluded from the differentiation algorithm by replacing it with zero, the algorithm becomes less robust and the probability of misclassification increases, as shown in Figure 13. In this case, when $\sigma_{A} \geq 0.02$, the performance of the classification is comparable to the performance of a randomized decision rule (Berger 1988), where $50 \%$ of the time the target is randomly guessed to be a plane, and $50 \%$ of the time it is guessed to be a corner, by completely ignoring the information carried by the data.

\subsection{Acute Corner Simulations}

Acute corners are less frequently encountered in comparison to the other target primitives. One example where they commonly occur is in orchestra shells for auditoriums and opera houses.

In the acute-corner simulations, the same sensing configuration as in the previous subsection is used. An acute corner with wedge angle $\theta_{c}$ is placed in front of the sensor pair at $r=2 \mathrm{~m}$, as shown in Figure 14. Each time a pulse is transmitted, four TOF and four amplitude measurements are collected. The stepper motor is rotated, and the target is scanned for $\theta$ from $-60^{\circ}$ to $60^{\circ}$. While obtaining classification results for each angular step, the unit scans the target from $\theta=-60^{\circ}$ to $\theta=60^{\circ}$, eight times. As a result, the logical sensing unit makes about 1,072 decisions for each pair of $\sigma_{t}$ and $\sigma_{A}$ values.

For the region in which an acute corner can be reliably differentiated with the classification algorithm $(\theta \in$ $\left.\left[-20^{\circ}, 20^{\circ}\right]\right)$, the results of belief assignments by a logical sensor unit for different values of $\theta_{c}$ are obtained, and the result for $\theta_{c}=60^{\circ}$ is provided in Figure 15 as an example. According to the results, for all $\theta_{c}$ values, the maximum belief of being an acute corner is obtained at $\theta=0^{\circ}$ when the system is noiseless. Moreover, the belief of being a plane or a corner is zero for all $\theta, \theta_{c}$, and $\sigma_{A}$ values. The values of $\sigma_{A}$ used in this study are 0.002 and 0.003 . Although the decrease in the belief of acute corner with increasing $|\theta|$ is sharper for larger $\theta_{c}$, the belief of acute corner is greater than the belief of unknown target for all $\theta$ and $\sigma_{A}$ values. Belief values are between 0.8 and 1.0 for $\theta_{c}=30^{\circ}$, between 0.7 and 1.0 for $\theta_{c}=45^{\circ}$, and between 0.6 and 1.0 for $\theta_{c}=60^{\circ}$.

The range, azimuth, and $\theta_{c}$ are estimated for acute corners with $\theta_{c}=30^{\circ}, 45^{\circ}$, and $60^{\circ}$ at $r=2 \mathrm{~m}$, for different $\sigma_{A}$ values. The results for $\sigma_{A}=0.002$ are provided in Figure 16 . For $\sigma_{A}=0.002$, the maximum range error is $5.7 \mathrm{~cm}$, and the maximum error in azimuth is $1.8^{\circ}$, which occurs with the acute corner of $\theta_{c}=30^{\circ}$, and the maximum error in $\theta_{c}$ is $1.4^{\circ}$, occurring for the acute corner of $\theta_{c}=60^{\circ}$.

The classification results for these acute corners are illustrated in Figure 17. In this figure, the probability of correct classification is higher than both the probability of misclassification and the probability of unknown target up to $\sigma_{t}=160 \mu \mathrm{sec}$ for $\theta_{c}=30^{\circ}, \sigma_{t}=100 \mu \mathrm{sec}$ for $\theta_{c}=45^{\circ}$, and $\sigma_{t}=40 \mu \mathrm{sec}$ for $\theta_{c}=60^{\circ}$. The probability of misclassification is always less than both the probability of correct classification and the probability of unknown target.

\section{Experimental Verification}

In this study, an experimental setup is employed to assign belief values to the experimentally obtained TOF and amplitude characteristics of the target primitives, and to test the proposed fusion method for target classification. Data was collected at Bilkent University Robotics and Sensing Research Laboratory. Three sensor nodes are placed in a small, uncluttered, rectangular room with specularly reflecting surfaces. Panasonic transducers are used, which have much wider beam width than the commonly used Polaroid transducers. The aperture radius of the Panasonic transducer is $a=0.65 \mathrm{~cm}$, and its resonant frequency is $f_{\mathrm{o}}=40 \mathrm{kHz}$; therefore $\theta_{\circ} \cong 54^{\circ}$ for these transducers (Panasonic 1989). Since Panasonic transducers are manufactured with distinct characteristics for transmitting and receiving, two transmitter/receiver pairs with very small vertical separation, as illustrated in Figure 18, are used as a single logical unit. The horizontal center-to-center separation between the transducers is $d=24.0 \mathrm{~cm}$. This sensing unit is mounted on a small 6-V stepper motor with step size $0.9^{\circ}$. The stepping action is controlled through the parallel port of an IBM-PC 486, with the aid of a microswitch. The sensor data is acquired using a DAS-50 A/D card with four channels, 12-bit resolution, and $1 \mathrm{MHz}$ sampling frequency. The echo signals are processed on an IBM-PC 486 using a $C$ language program. From the time of transmission, 10,000 samples of each echo signal are collected and thresholded. The amplitude information is extracted by finding the maximum value of the signal after the threshold value is exceeded. The targets employed in this study are: cylinders with radii $1.5,2.5,5.0$, and $7.5 \mathrm{~cm}$; a planar target; a corner; and an acute corner of $\theta_{c}=60^{\circ}$.

All of the experiments are conducted on large sheets of millimetric paper to allow accurate calibration. In the experiments, each target's surface distance $r$ to the center of the transducer system is varied between $20 \mathrm{~cm}$ to $140 \mathrm{~cm}$ at 10 $\mathrm{cm}$ intervals. At each position, the target is scanned while it is stationary at $\theta=0^{\circ}$. The typical differential TOF between the transducers varies between $0 \mathrm{~cm}$ and $14 \mathrm{~cm}$, depending on the target type, curvature, and distance for the fixed separation of $d=24.0 \mathrm{~cm}$ (Ayrulu 1996). As the range of the target increases, the differential signal becomes less reliable for target classification.

Belief-assignment results to the TOF and amplitude characteristics of a plane at $r=50 \mathrm{~cm}$ when scanned with the sensing unit are given in Figure 19. In this figure, belief of being a planar target primitive is greater than zero for 


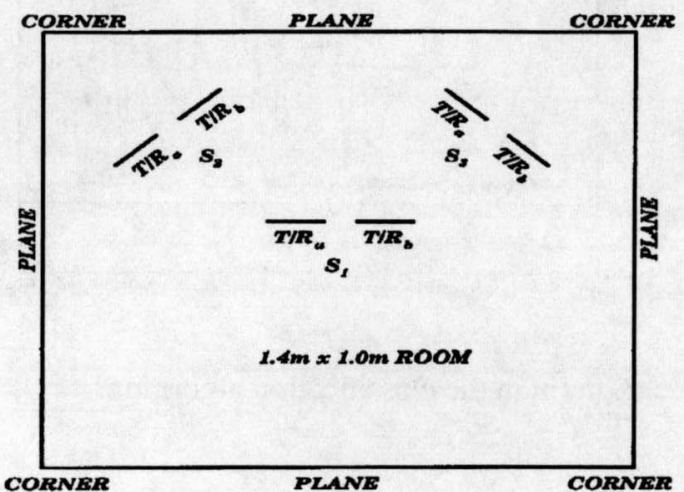

(a)

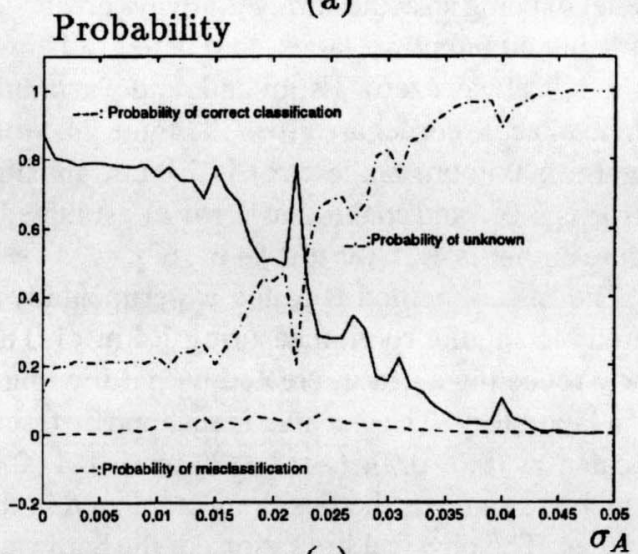

(c)

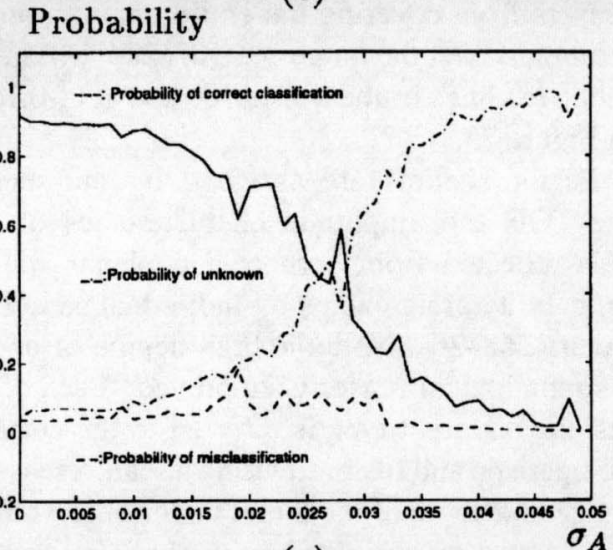

(e)

\section{Probability}

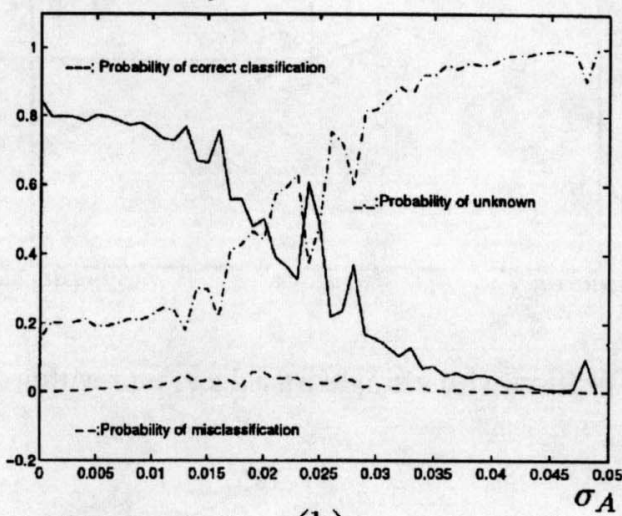

(b)

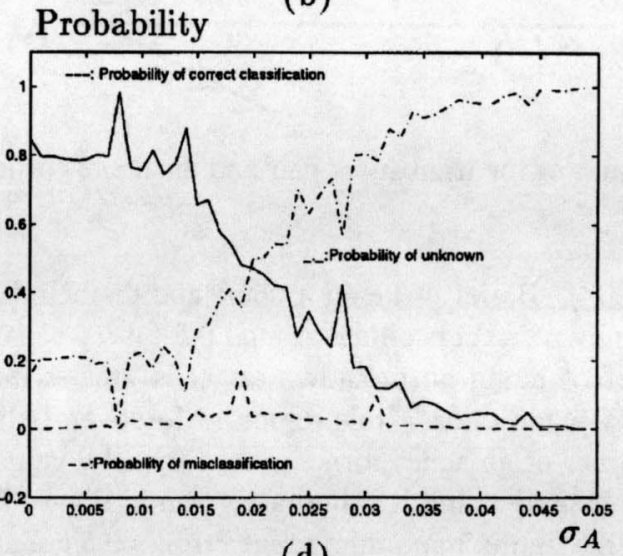

(d)

\section{Probability}

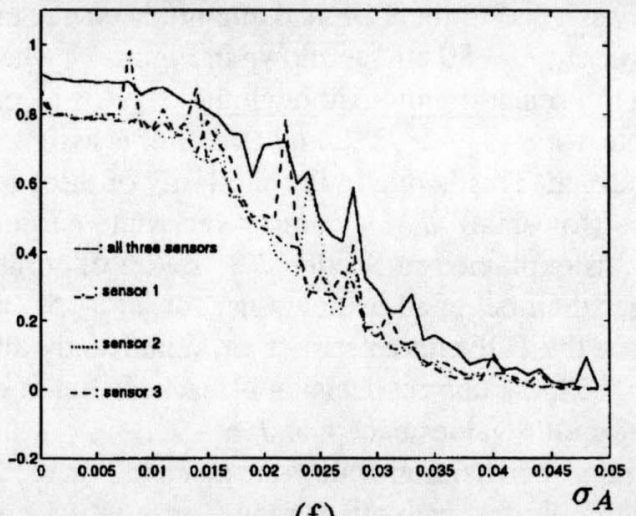

(f)

Fig. 12. (a) The simulated room; (b) classification results: sensor at $(0.0,0.0)$; (c) sensor at $(-0.1,0.1)$; (d) sensor at $(0.1$, 0.1 ); (e) all three sensors; (f) improvement in the probability of correct classification. 

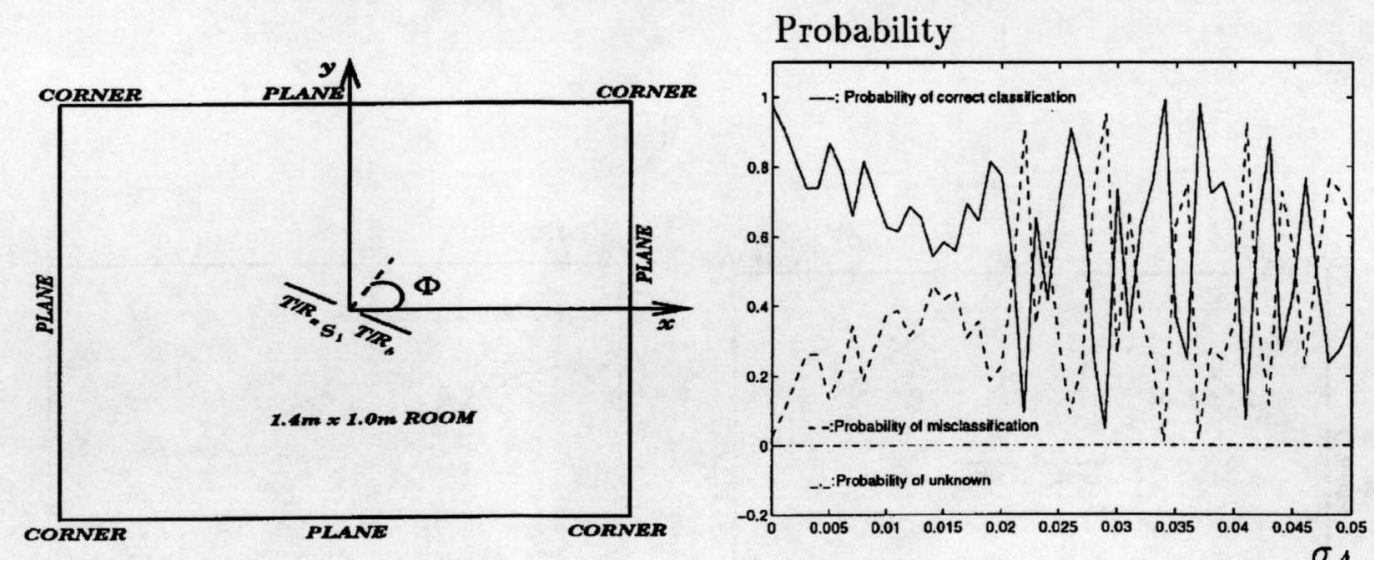

Fig. 13. Classification with a single transducer pair without the $\sigma_{A}$ term in the classification algorithm.

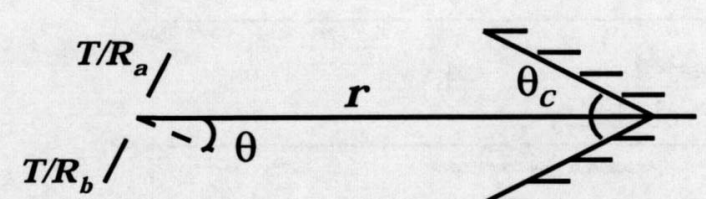

Fig. 14. Position of the transducer pair and the acute corner.

$\theta \in\left[-20^{\circ}, 20^{\circ}\right]$. Belief of being a plane and the belief of being an unknown target oscillate around 0.5 for $|\theta| \leq 10^{\circ}$, and the belief of being an unknown target is greater than the belief of a plane outside this region. Moreover, belief of being a corner or an acute corner is zero for all $\theta$ values. Estimated range and azimuth values are given in Figure 20. Referring to this figure, maximum range error is $0.5 \mathrm{~cm}$ and maximum error in the azimuth estimate is $0.7^{\circ}$.

Beliefs are assigned to the TOF and amplitude characteristics of a corner at $r=80 \mathrm{~cm}$, as shown in Figure 21 when scanned with the sensing unit. Although the target is a corner, for the interval $\theta \in\left[-5^{\circ}, 2^{\circ}\right]$, highest belief is assigned to the acute corner. This is due to the similarity of the TOF characteristics (for small $|\theta|$ ) of corners and acute corners with large $\theta_{c}$, as explained in Section 2.2. Belief of corner becomes larger than belief of acute corner for $|\theta| \geq 5^{\circ}$, as expected. Since the TOF characteristics are significantly different for $|\theta| \geq 5^{\circ}$, the correct decision is reached. Belief of plane is zero for all $\theta$ values except at $\theta=-9^{\circ}$.

Estimated range and azimuth values are given in Figure 22. Referring to this figure, maximum range error is $0.3 \mathrm{~cm}$, and the maximum error in azimuth is $3.6^{\circ}$ in the region $\theta \in\left[-4^{\circ}, 4^{\circ}\right]$. In Figure 22c, estimated wedge angle of the acute corner is shown. Although the belief for an acute corner is around one for $|\theta| \leq 5^{\circ}$, estimated wedge angle is around $90^{\circ}$ in this region. Therefore, the final decision is a corner, as discussed in Section 2.2.

Beliefs assigned to the TOF and amplitude characteristics of an acute corner of $\theta_{c}=60^{\circ}$ at $r=40 \mathrm{~cm}$, which is scanned with the same system, are given in Figure 23. In this figure, belief of being an acute corner is always greater than the belief of being an unknown target, and belief of being a plane or a corner is always zero. Estimated range, azimuth, and wedge angle of acute corner are given in Figure 24. Referring to this figure, maximum range error is $2.0 \mathrm{~cm}$, maximum azimuth error is $3.0^{\circ}$, and maximum error in estimated angle of the acute corner is $4.2^{\circ}$ for $\theta \in\left[-6^{\circ}, 6^{\circ}\right]$.

The fusion method is tested experimentally in an uncluttered rectangular room measuring $1.4 \mathrm{~m} \times 1.0 \mathrm{~m}$ with specularly reflecting surface, created by partitioning off a section of a laboratory. The test area is scanned by three sensor units located at $(0.0,0.0),(-0.1,0.1)$, and $(0.1,0.1)$ in meters, which are same as the positions employed in the simulation studies. The physical limitations of the hardware prevent the sensors from covering the entire angular range $\phi$. Instead, rotation is over the range $\phi \in\left[0^{\circ}, 284^{\circ}\right]$. As an example, the range readings of the sensor located at $(-0.1,0.1)$ are given in Figure 25.

Feature beliefs are assigned by the sensors based on the TOF and amplitude characteristics of the sonar signals reflected from corners and planar walls. The basic probability assignments by individual sensors are shown in Figure 26a-26c. Note the high degree of uncertainty, since a single logical sensor is employed. Each of the sensor decisions on target type is referred to the central position for comparison and fusion. During a scan, a sensor estimates the range and angle of the target under observation. The values for a target are weighted by the beliefs assigned to the estimates, and then referred to position $(0.0,0.0)$. The sensors' determinations of beliefs are fused using Dempster's rule of combination. Fusion results are shown in Figure 26d. Using a single sensing node, the percentage of correct decisions is about $30 \%$. The remaining $70 \%$ is attributed to incorrect decisions due to noise and complete uncertainty, which occurs when the target is not visible to the sensor at certain viewpoints during a scan. When decisions of two nodes are 

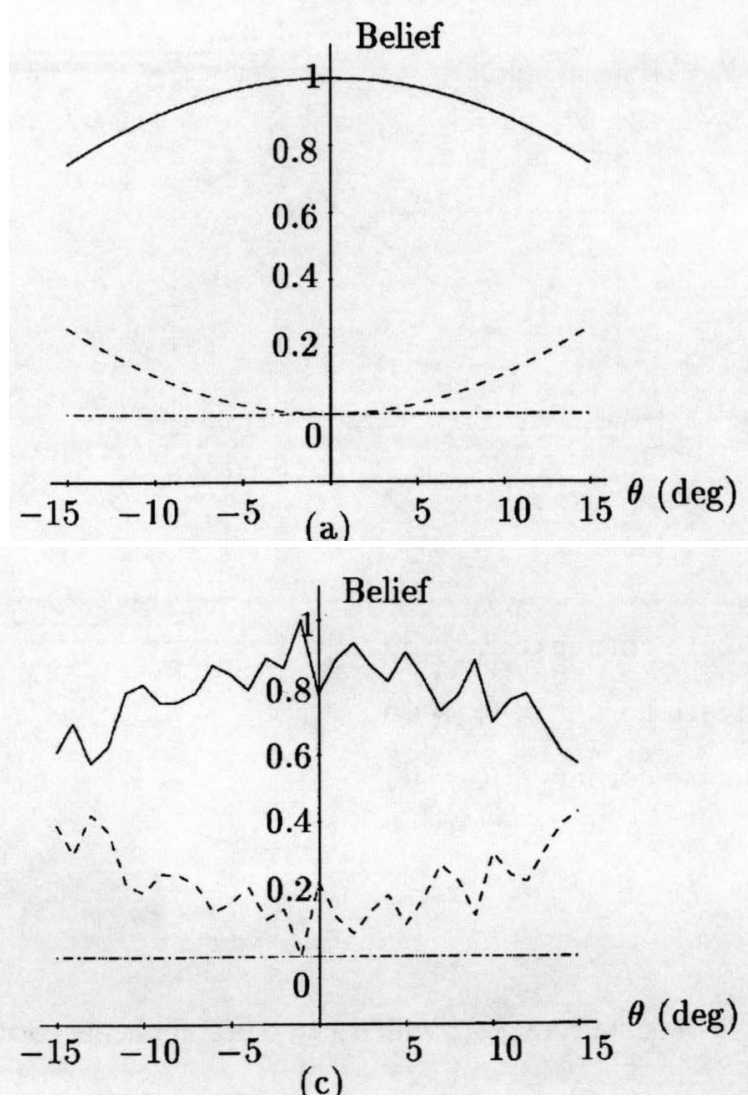

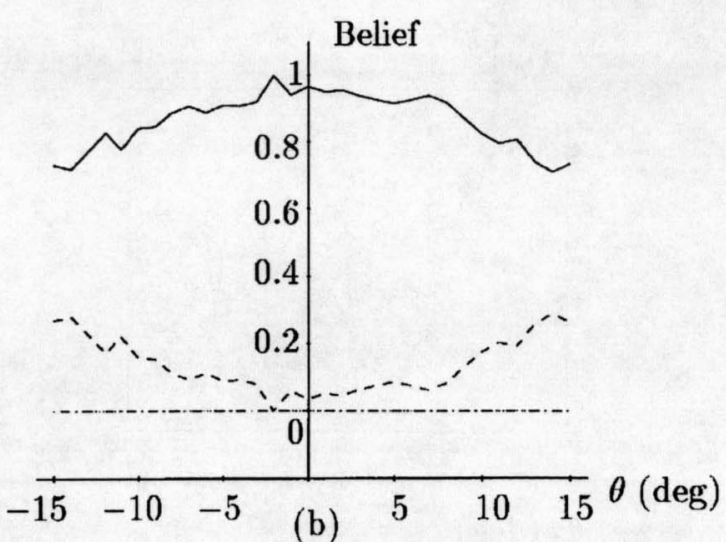

-

-..-..: belief of corner, belief of plane

- . - - belief of unknown

Fig. 15. Belief assignment by the sensing unit when an acute corner at $r=2 \mathrm{~m}$ with $\theta_{c}=60^{\circ}$ is scanned. Results for (a) $\sigma_{A}=0.0$; (b) $\sigma_{A}=0.002$; and (c) $\sigma_{A}=0.003$.

fused using the Dempster's rule, the correct decision percentage improves to $43.6 \%$. This percentage is $51.9 \%$ when the decisions of three nodes are fused. A navigation algorithm would apply a belief threshold to these values to reliably recognize targets with belief above a set threshold. The accuracy of the map improves with the number of sensor nodes used. In addition, there is a trade-off between the threshold level versus false target recognition. The higher the threshold, the lower the false recognition rate. On the other hand, some targets that are difficult to detect, such as edges and thin cylinders, may not be detected if the threshold is set too high. After fusion over three sensing nodes, a threshold level between 0.3 and 0.5 is a good choice. In fact, 0.5 is approximately equal to the correct decision rate with three sensing nodes.

\section{Conclusion}

This work presents a novel application of the theory of evidence for target or beacon recognition. Physical models are used to model reflections from target primitives commonly encountered in mobile-robot applications. Target features are generated as being evidentially tied to degrees of belief which are subsequently fused for multiple sonars at distinct geographical sites. Using both TOF and amplitude data in the feature fusion process allows more robust differentiation.

Employing an evidential reasoning approach in the differentiation process enables the modeling of nonparametric uncertainty. Fusion of feature data from multiple sensors using Dempster's rule of combination reduces such perception uncertainty. The consequent increase in processing time does not entail a significant cost, considering the speeds of modern computers. Although this paper reports the use of only three sensing nodes, it has been experimentally demonstrated elsewhere that the methodology is suitable for real-time applications with up to 15 sensing sites (Ayrulu, Barshan, and Utete 1997; Utete, Barshan, and Ayrulu Forthcoming).

The results have ground for application in mobile robotics, where multiple sensing agents or robots are employed to survey an unknown environment composed of primitive target types. As for future work, the proposed fusion method can be extended to include physically different sensors, such as infrared and laser-ranging systems for map building, target identification, localization, and tracking applications. The eventual goal is to realize robots capable of a high degree of 

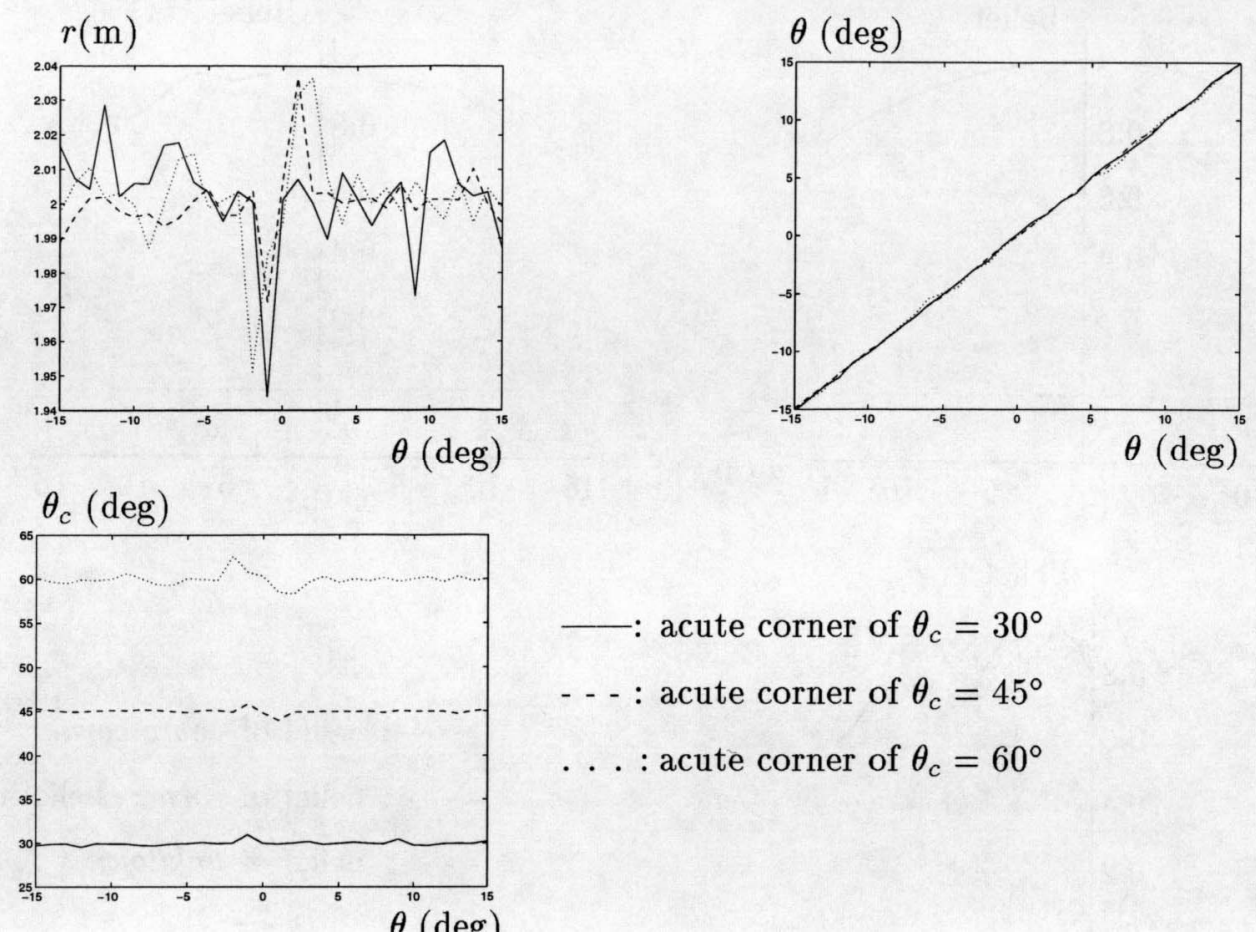

— : acute corner of $\theta_{c}=30^{\circ}$

- - : acute corner of $\theta_{c}=45^{\circ}$

. . : : acute corner of $\theta_{c}=60^{\circ}$

Fig. 16. Estimated values of range $r$, inclination angle $\theta$, and angle of acute corner $\theta_{c}$, with $\sigma_{\mathcal{A}}=0.002$ for acute corners of $\theta_{c}=30^{\circ}, 45^{\circ}$, and $60^{\circ}$ at $r=2 \mathrm{~m}$.

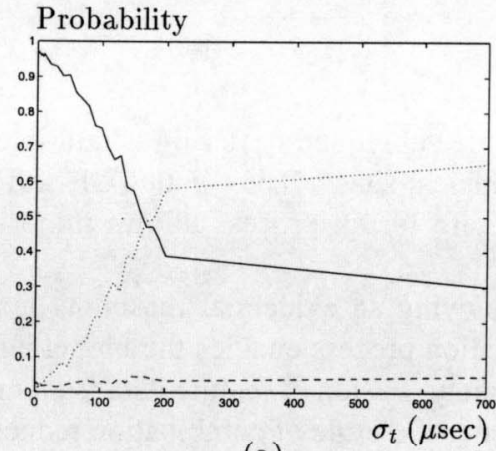

(a)

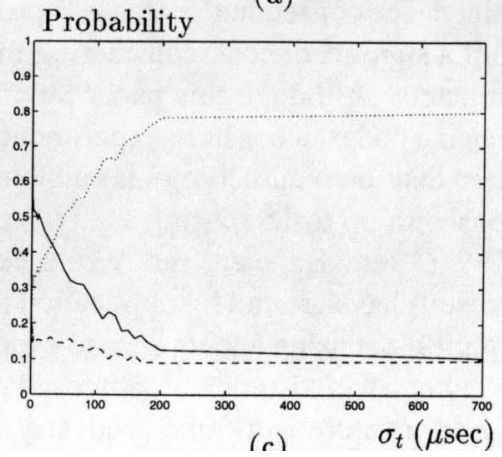

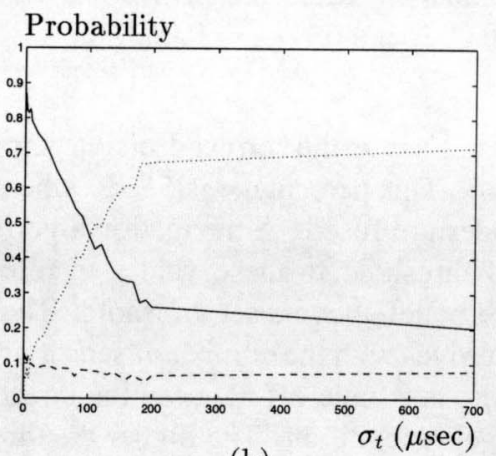

(b)

$\longrightarrow$ : probability of true classification

- . : probability of misclassification

$\ldots$ : probability of unknown

Fig. 17. Classification results with a single sensing unit when acute corners at $r=2 \mathrm{~m}$ with (a) $\theta_{c}=30^{\circ}$; (b) $\theta_{c}=45^{\circ}$; (c) $\theta_{c}=60^{\circ}$ are scanned. 


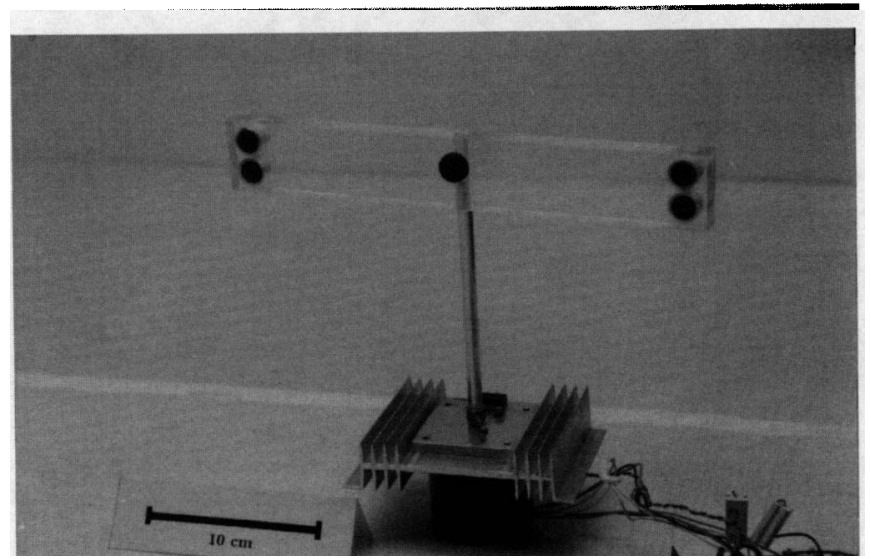

Fig. 18. Configuration of the Panasonic transducers in the real system.

autonomy through the use of multiple sensors. The work can be further generalized to three-dimensional targets. Coordination of the sensing agents and strategic target recognition while either or both the sensors and the targets are in motion is another possible direction for future research.

\section{Acknowledgments}

This research was supported by TÜBİTAK, under grants EEEAG-92 and EEEAG-116. The authors would like to thank Dr. Aydan M. Erkmen and Dr. İsmet Erkmen of Middle East Technical University and Dr. Simukai W. Utete of the University of Oxford for many useful discussions and comments, and the anonymous reviewers for their comments and suggestions.

\section{Appendix: \\ Geometric Target and Echo Signal Models}

In this appendix, geometric models of the target primitives used in this study are provided. The targets modeled are plane, corner, acute corner, edge, and cylinder. For each target primitive, models of the corresponding echo signals are given, which are based on experimental data.

\section{Planar Target Model}

A plane has a line segment profile in 2-D. The geometry of reflection for a planar target is illustrated in Figure 27. Since each transducer can be employed both as transmitter and receiver, a set of four TOF and four amplitude measurements are obtained each time data is collected from the target. From the geometry, the TOF measurements for each transmitter and receiver pair are found as

$$
t_{a a}=\frac{2 r_{a}}{c}
$$

$$
\begin{aligned}
t_{a b}=t_{b a} & =\frac{r_{1}+r_{2}}{c}, \text { and } \\
t_{b b} & =\frac{2 r_{b}}{c},
\end{aligned}
$$

where $t_{a b}$ denotes TOF extracted from $A_{a b}(r, \theta, d, t)$, which is the signal transmitted by $a$ and received by $b$ at time $t$. In earlier work, it has been shown that the reflected signals are well approximated by a sinusoid with a Gaussian envelope (Kuc and Siegel 1987). The detected signals by each transmitter and receiver pair can be modeled as

$$
\begin{aligned}
A_{a a}(r, \theta, d, t) & =A_{\max } \frac{r_{\min }}{r_{a}} e^{-\frac{\theta^{2}}{\sigma_{T}^{2}}} e^{-\frac{\left(t-t_{a a}-\frac{3}{f_{0}}\right)^{2}}{\sigma^{2}}} \\
& \times \sin \left[2 \pi f_{\circ}\left(t-t_{a a}\right)\right] \\
A_{a b}(r, \theta, d, t) & =A_{\max } \frac{2 r_{\min }}{r_{1}+r_{2}} e^{-\frac{\theta_{1}^{2}+\theta_{2}^{2}}{2 \sigma_{T}^{2}}} e^{-\frac{\left(t-t_{a b}-\frac{3}{t_{0}}\right)^{2}}{\sigma^{2}}} \\
& \times \sin \left[2 \pi f_{\circ}\left(t-t_{a b}\right)\right] \\
A_{b a}(r, \theta, d, t) & =A_{\max } \frac{2 r_{\min }}{r_{2}+r_{1}} e^{-\frac{\theta_{2}^{2}+\theta_{1}^{2}}{2 \sigma_{T}^{2}}} e^{-\frac{\left(t-t_{b a}-\frac{3}{t_{0}}\right)^{2}}{\sigma^{2}}} \\
& \times \sin \left[2 \pi f_{0}\left(t-t_{b a}\right)\right], \text { and } \\
A_{b b}(r, \theta, d, t) & =A_{\max } \frac{r_{\min }}{r_{b}} e^{-\frac{\theta^{2}}{\sigma_{T}^{2}}} e^{-\frac{\left(t-t_{b b}-\frac{3}{t_{0}}\right)^{2}}{\sigma^{2}}} \\
& \times \sin \left[2 \pi f_{\circ}\left(t-t_{b b}\right)\right]
\end{aligned}
$$

In all of the signal models, $\sigma_{T}$ is chosen as $\frac{\theta_{0}}{2}$ (Bozma 1992). The form of the range-attenuation term $r_{1}+r_{2}$ in eqs. (66) and (67) is due to the specular reflection of the beam. Since $r_{\min }$ is the minimum distance at which the beam-pattern model described above is valid, the beam will be in the near zone when $r<r_{\text {min }}$, where the far-zone beam model cannot be used anymore. According to the piston 
model of the transducer (Zemanek 1971), in the near zone, the beam is confined to a cylinder with radius $a$, which is the radius of the transducer. The length of this zone is approximately $\frac{\left(4 a^{2}-\lambda^{2}\right)}{4 \lambda}$ from the face of the transducer where $\lambda$ is the wavelength of the acoustic transmission. On the other hand, the beam is confined to a cone with angle $2 \theta_{0}$ in the far zone.

\section{Corner-Target Model}

A right-angle corner is the line between two perpendicular planes that form a concave dihedral, as illustrated in Figure 28. When the target is a corner, the received signal consists of two components: the diffracted signal that originates from the junction of the two planes; and the doubly reflected signal from the two intersecting planes. The diffracted signal can be neglected, since its contribution to amplitude is much smaller than that of the reflected signal. The TOF measurements for each transmitter can be found as

$$
\begin{aligned}
t_{a a} & =\frac{2 r_{a}}{c}, \\
t_{a b}=t_{b a} & =\frac{2 r}{c}, \text { and } \\
t_{b b} & =\frac{2 r_{b}}{c} .
\end{aligned}
$$

The sonar signal reflected from a corner target can be modeled as

$$
\begin{aligned}
A_{a a}(r, \theta, d, t) & =A_{\max } \frac{r_{\min }}{r_{a}} e^{-\frac{\theta_{a}^{2}}{\sigma_{T}^{2}}} e^{-\frac{\left(t-t_{a a}-\frac{3}{t_{0}}\right)^{2}}{\sigma^{2}}} \\
& \times \sin \left[2 \pi f_{\circ}\left(t-t_{a a}\right)\right], \\
A_{a b}(r, \theta, d, t) & =A_{\max } \frac{r_{\min }}{r} e^{-\frac{\theta^{2}}{\sigma_{T}^{2}}} e^{-\frac{\left(t-t_{a b}-\frac{3}{f_{0}}\right)^{2}}{\sigma^{2}}} \\
& \times \sin \left[2 \pi f_{\circ}\left(t-t_{a b}\right)\right], \\
A_{b a}(r, \theta, d, t) & =A_{\max } \frac{r_{\min }}{r} e^{-\frac{\theta^{2}}{\sigma_{T}^{2}}} e^{-\frac{\left(t-t_{b a}-\frac{3}{f_{0}}\right)^{2}}{\sigma^{2}}} \\
& \times \sin \left[2 \pi f_{\circ}\left(t-t_{b a}\right)\right], \text { and } \\
A_{b b}(r, \theta, d, t) & =A_{\max } \frac{r_{\min }}{r_{b}} e^{-\frac{\theta_{b}^{2}}{\sigma_{T}^{2}}} e^{-\frac{\left(t-t_{b b}-\frac{3}{f_{0}}\right)^{2}}{\sigma^{2}}} \\
& \times \sin \left[2 \pi f_{\circ}\left(t-t_{b b}\right)\right] .
\end{aligned}
$$

\section{Edge-Target Model}

An edge is the intersection of two planes that form a convex space when observed from the transducers. This target type is illustrated in Figure 29. For the edge, the acoustic signal diffracts and spreads out cylindrically once it reaches the line defining the edge. Owing to the relatively lower echo amplitude, edges are the most difficult and problematic targets to detect in a mobile robot's environment. For the edge, the TOF measurements of each transmitter and receiver pair can be found as

$$
\begin{aligned}
t_{a a} & =\frac{2 r_{a}}{c}, \\
t_{a b}=t_{b a} & =\frac{r_{a}+r_{b}}{c}, \text { and } \\
t_{b b} & =\frac{2 r_{b}}{c} .
\end{aligned}
$$

The signal waveforms are modeled as

$$
\begin{aligned}
A_{a a}(r, \theta, d, t) & =\rho_{e} \frac{A_{\max } r_{\min }^{3 / 2}}{r_{a}^{3 / 2}} e^{-\frac{\theta_{a}^{2}}{\sigma_{T}^{2}}} e^{-\frac{\left(t-t_{a a}-\frac{3}{f_{0}}\right)^{2}}{\sigma^{2}}} \\
& \times \sin \left[2 \pi f_{0}\left(t-t_{a a}\right)\right], \\
A_{a b}(r, \theta, d, t) & =\rho_{e} \frac{A_{\max } r_{\min }^{3 / 2}}{r_{a} r_{b}^{1 / 2}} e^{-\frac{\theta_{a}^{2}+\theta_{b}^{2}}{2 \sigma_{T}^{2}}} e^{-\frac{\left(t-t_{a b}-\frac{3}{t_{0}}\right)^{2}}{\sigma^{2}}} \\
& \times \sin \left[2 \pi f_{\mathrm{o}}\left(t-t_{a b}\right)\right], \\
A_{b a}(r, \theta, d, t) & =\rho_{e} \frac{A_{\max } r_{\min }^{3 / 2}}{r_{b} r_{a}^{1 / 2}} e^{-\frac{\theta_{b}^{2}+\theta_{a}^{2}}{2 \sigma_{T}^{2}}} e^{-\frac{\left(t-t_{b a}-\frac{3}{f_{0}}\right)^{2}}{\sigma^{2}}} \\
& \times \sin \left[2 \pi f_{0}\left(t-t_{b a}\right)\right], \text { and } \\
A_{b b}(r, \theta, d, t) & =\rho_{e} \frac{A_{\max } r_{\min }^{3 / 2}}{r_{b}^{3 / 2}} e^{-\frac{\theta_{b}^{2}}{\sigma_{T}^{2}}} e^{-\frac{\left(t-t_{b b}-\frac{3}{f_{0}}\right)^{2}}{\sigma^{2}}} \\
& \times \sin \left[2 \pi f_{\mathrm{o}}\left(t-t_{b b}\right)\right],
\end{aligned}
$$

where $\rho_{e}$ is a reflection coefficient that decreases with $\theta_{e}$, defining the sharpness of the wedge. Based on experimental data (Kuc and Barshan 1989), $\rho_{e}$ can be approximately modeled as a linear function of $\theta_{e}$, which is the angle of the edge $\left(\theta_{e} \leq \frac{\pi}{2}\right)$ given in Figure 29 such that

$$
\rho_{e} \frac{A_{\max } r_{\min }^{3 / 2}}{r_{a}^{3 / 2}}=0.05 \text { if } \theta_{e}=90^{\circ}
$$

and

$$
\rho_{e} \frac{A_{\max } r_{\min }^{3 / 2}}{r_{a}^{3 / 2}}=0.025 \text { if } \theta_{e}=60^{\circ},
$$

where $A_{\max }=1, r_{\min }=10 \mathrm{~cm}$, and $r_{a} \cong 10 \mathrm{~cm}$. Then, $\rho_{e}$ can be found as

$$
\rho_{e}=8.43 \times 10^{-3} \theta_{e}-0.25298,
$$

with $\theta_{e}$ in degrees.

\section{Cylinder-Target Model}

Although not as common as planes and corners, a mobile robot often comes across a cylindrical target in its environment in the form of pillars or poles. The geometry of reflections from this target type is illustrated in Figure 30. Experimental observations indicate that for a cylinder with 


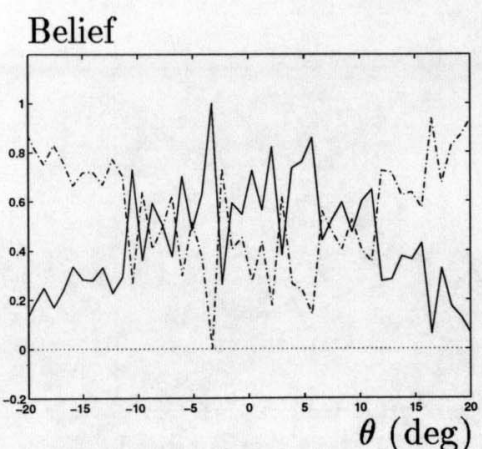

- -: belief of plane

.....: belief of corner, belief of acute corner

..--- belief of unknown

Fig. 19. Belief assignment to a plane at $r=50 \mathrm{~cm}$, scanned with the logical sensing unit.

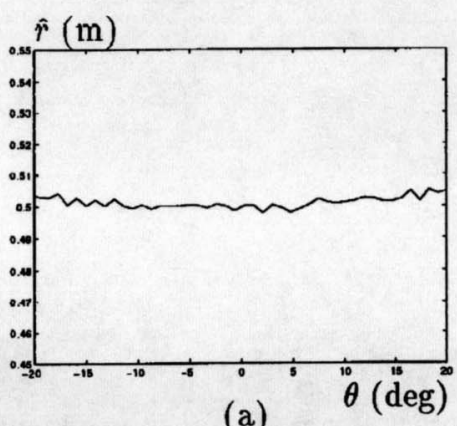

(a)

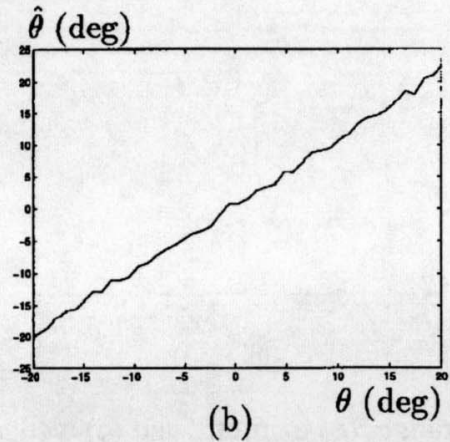

(b)

Fig. 20. Estimated (a) range and (b) azimuth values of a plane at $r=50 \mathrm{~cm}$, scanned with the sensing unit.

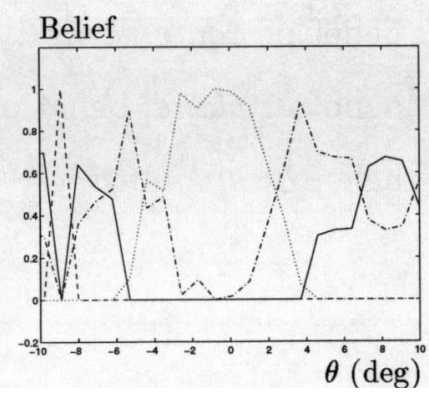

—: belief of corner

- - : belief of plane

.....: belief of acute corner

-..--: belief of unknown

Fig. 21. Belief assignment to a corner at $r=80 \mathrm{~cm}$, scanned with the sensing unit.

radius $2.54 \mathrm{~cm}$ located at $10 \mathrm{~cm}$, the echo amplitude is observed to be $50 \%$ of that from a normally incident plane at the same range (Kuc and Barshan 1989). The TOF measurements and signal models of each transmitter and receiver pair are

$$
\begin{aligned}
t_{a a} & =\frac{2 r_{a}}{c}, \\
t_{a b}=t_{b a} & =\frac{r_{1}+r_{2}}{c}, \text { and } \\
t_{b b} & =\frac{2 r_{b}}{c} .
\end{aligned}
$$

$$
\begin{aligned}
A_{a a}(r, \theta, d, t) & =\rho_{c} \frac{A_{\max } r_{\min }^{3 / 2}}{r_{a}^{3 / 2}} e^{-\frac{\theta_{a}^{2}}{\sigma_{T}^{2}}} e^{-\frac{\left(t-t_{a a}-\frac{3}{f_{0}}\right)^{2}}{\sigma^{2}}} \\
& \times \sin \left[2 \pi f_{\mathrm{o}}\left(t-t_{a a}\right)\right], \\
A_{a b}(r, \theta, d, t) & =\rho_{c} \frac{A_{\max } r_{\min }^{3 / 2}}{r_{1} r_{2}^{1 / 2}} e^{-\frac{\theta_{1}^{2}+\theta_{2}^{2}}{2 \sigma_{T}^{2}}} e^{-\frac{\left(t-t_{a b}-\frac{3}{f_{0}}\right)^{2}}{\sigma^{2}}} \\
& \times \sin \left[2 \pi f_{\mathrm{o}}\left(t-t_{a b}\right)\right], \\
A_{b a}(r, \theta, d, t) & =\rho_{c} \frac{A_{\max } r_{\min }^{3 / 2}}{r_{2} r_{1}^{1 / 2}} e^{-\frac{\theta_{2}^{2}+\theta_{1}^{2}}{2 \sigma_{T}^{2}}} e^{-\frac{\left(t-t_{b a}-\frac{3}{f_{0}}\right)^{2}}{\sigma^{2}}}
\end{aligned}
$$



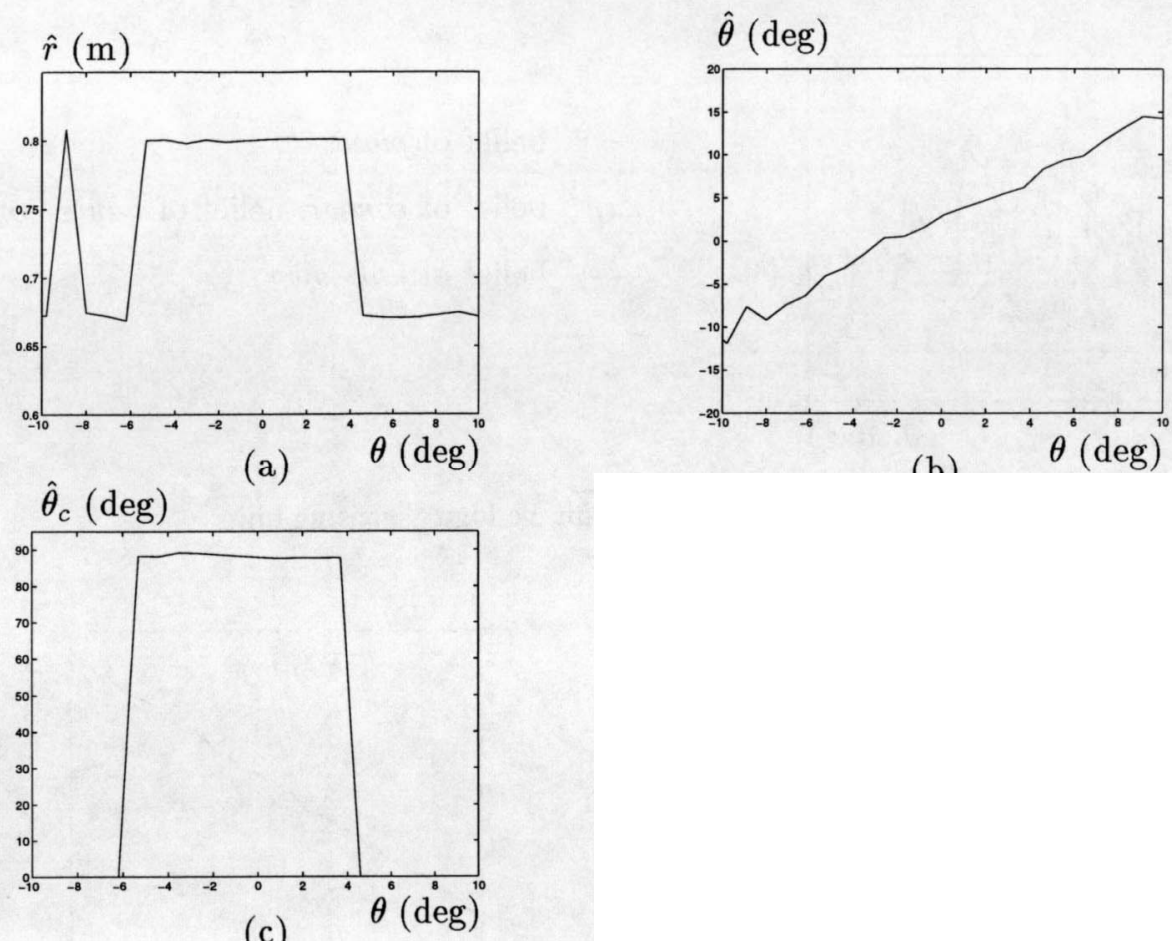

(h)

Fig. 22. Estimated (a) range, (b) azimuth, and (c) wedge angle of a corner at $r=80 \mathrm{~cm}$, scanned with the sensing unit.

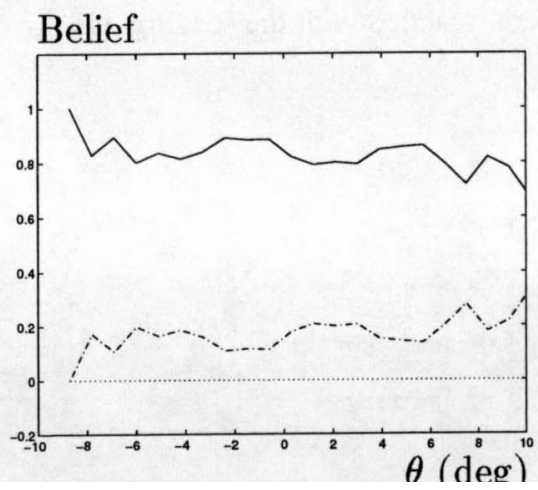

—-: belief of acute corner

.....: belief of plane, belief of corner

-.-.-: belief of unknown

Fig. 23. Belief assignment to an acute corner with $\theta_{c}=60^{\circ}$ at $r=40 \mathrm{~cm}$, scanned with the sensing unit.

$$
\begin{aligned}
& \times \sin \left[2 \pi f_{0}\left(t-t_{b a}\right)\right], \text { and } \\
A_{b b}(r, \theta, d, t) & =\rho_{c} \frac{A_{\max } r_{\min }^{3 / 2}}{r_{b}^{3 / 2}} e^{-\frac{\theta_{b}^{2}}{\sigma_{T}^{2}}} e^{-\frac{\left(t-t_{b b}-\frac{3}{t_{0}}\right)^{2}}{\sigma^{2}}} \\
& \times \sin \left[2 \pi f_{0}\left(t-t_{b b}\right)\right],
\end{aligned}
$$

where $\rho_{c}$ is the reflection coefficient of the cylinder, which depends on its radius of curvature. When the radius of the cylinder increases, the amplitude of the detected signal increases. An approximate model is to vary $\rho_{c}$ linearly with the radius of the cylinder based on the given data points. A better model would result if more data points were taken and the best-fitting curve was found. In the upper limit, when $r_{c}$ is larger than $10 \mathrm{~m}$, the cylinder behaves like a planar target

According to the amplitude curves in Figure 31 (obtained by using the above models), for $r_{c} \geq 10 \mathrm{~m}$ the amplitude curves of the transmitter and receiver pairs are the same as the curves obtained when the target is a plane. As a result,

$$
\lim _{r_{c} \rightarrow \infty} \rho_{c} \frac{A_{\max } r_{\min }^{3 / 2}}{r_{a}^{3 / 2}}=\frac{A_{\max } r_{\min }}{r_{a}}
$$

In the limit as $r_{c}$ goes to zero, a line target is obtained. In this case, if $r_{c} \leq 5 \mathrm{~cm}$, the cylinder behaves like an edgetype target with $\theta_{e} \leq 30^{\circ}$. Then, $\rho_{c} \cong \rho_{e}$. Using eq. (83), $\rho_{e}$ 

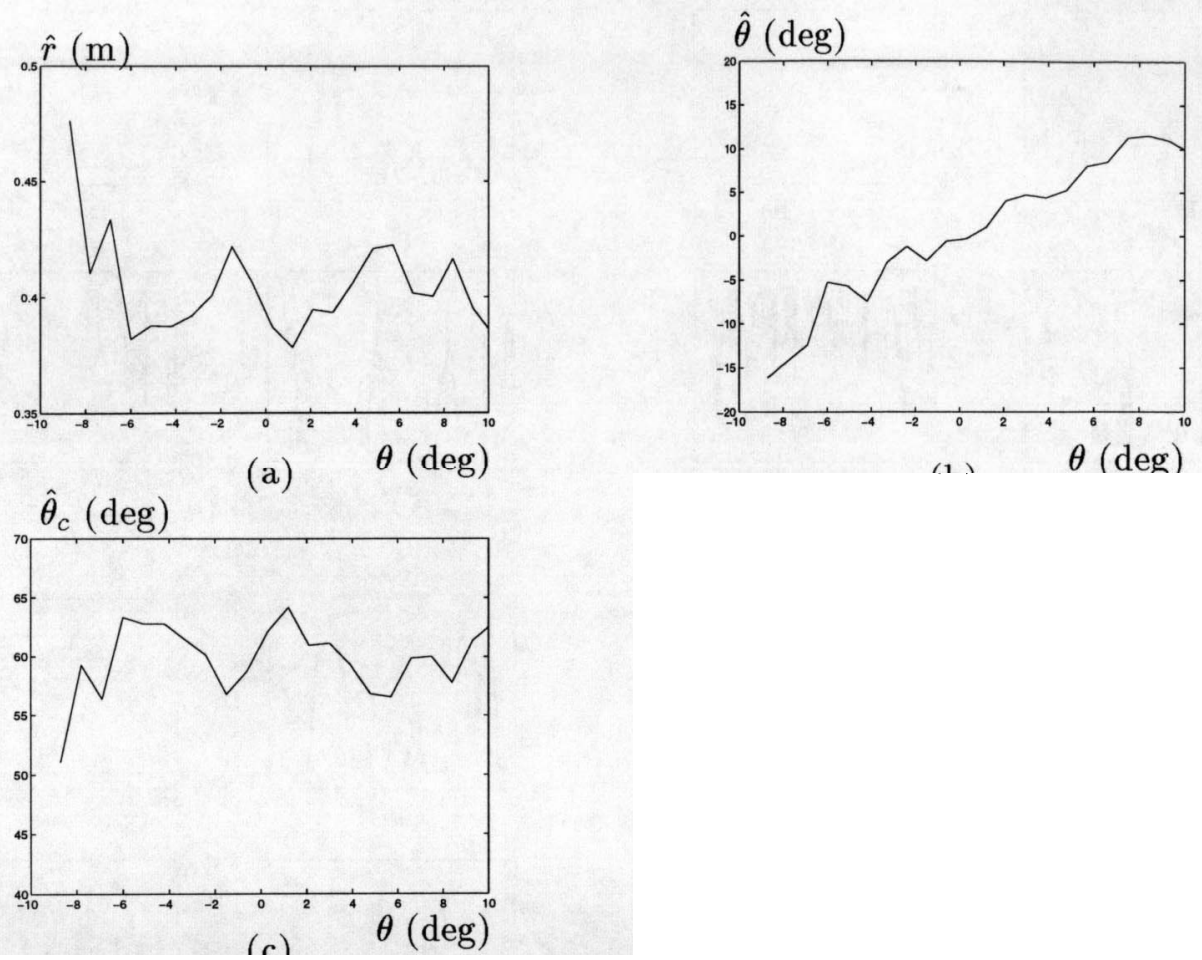

Fig. 24. Estimated (a) range, (b) azimuth, and (c) wedge angle of an acute corner of $\theta_{c}=60^{\circ}$ at $r=40 \mathrm{~cm}$, scanned with the sensing unit.

is calculated as $1.98 \times 10^{-6}$ for $\theta_{e}=30^{\circ}$. Hence the linear dependence between $\rho_{c}$ and $r_{c}$ is

$$
\rho_{c}=0.44946 r_{c}-0.022471
$$

where $r_{c}$ is in meters.

Moreover, it can be seen from the equations of the detected signals for both the edge and the cylinder that they produce a cylindrically divergent echo wavefront that decays as $r^{-1 / 2}$. In both cases, the received signal is only composed of the diffracted signal. Since this diffracted component attenuates very quickly, reflections from edges and cylinders with small cross sections are the most difficult echoes to detect.

\section{Acute-Corner Model}

An acute corner is an intersection of two planes that form a concave space when observed from the transducers. Wedge angle $\theta_{c}$ of an acute corner varies from 0 to $90^{\circ}$. From the geometry of Figure 32, the measurements can be expressed as

$$
\begin{aligned}
r_{a a} & =2 r_{a} \sin \theta_{c}, \\
r_{b b} & =2 r_{b} \sin \theta_{c}, \text { and } \\
r_{a b} & =\sqrt{r_{a}^{2}+r_{b}^{2}-2 r_{a} r_{b} \cos \left(2 \theta_{c}-\phi_{T}\right)} .
\end{aligned}
$$

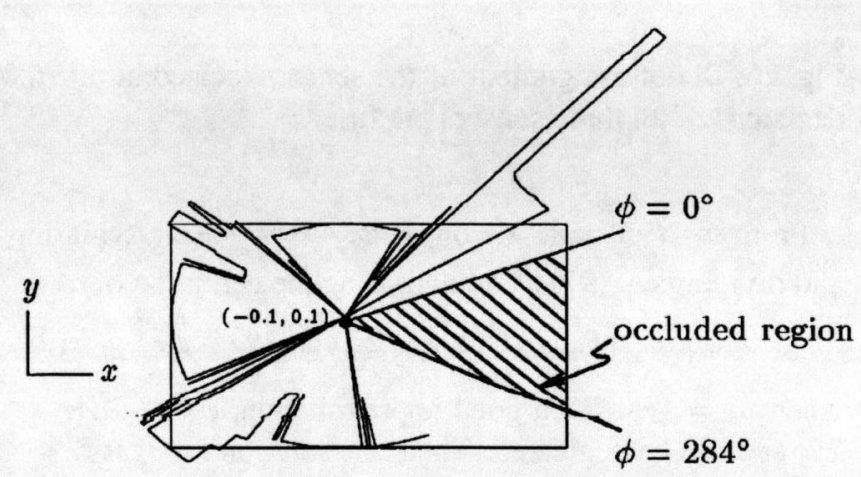

Fig. 25. Range readings of the sensor located at $(-0.1,0.1)$ in a rectangular room.

Note that the round-trip distances measured by sensors $a$ and $b$ are twice their distance from the target when the target is a $90^{\circ}$ corner, but are less than their actual distance by a factor of $\sin \theta_{c}$ if the target is an acute corner with wedge angle $\theta_{c}$. The $\sin \theta_{c}$ factor can be observed in Figure 5, where at fixed distance to the target, the measured TOF increases with increasing values of $\theta_{c}$. The observation points that give the same TOF reading fall onto an arc bounded by the acute corner, with the center of the arc being at the junction point. 

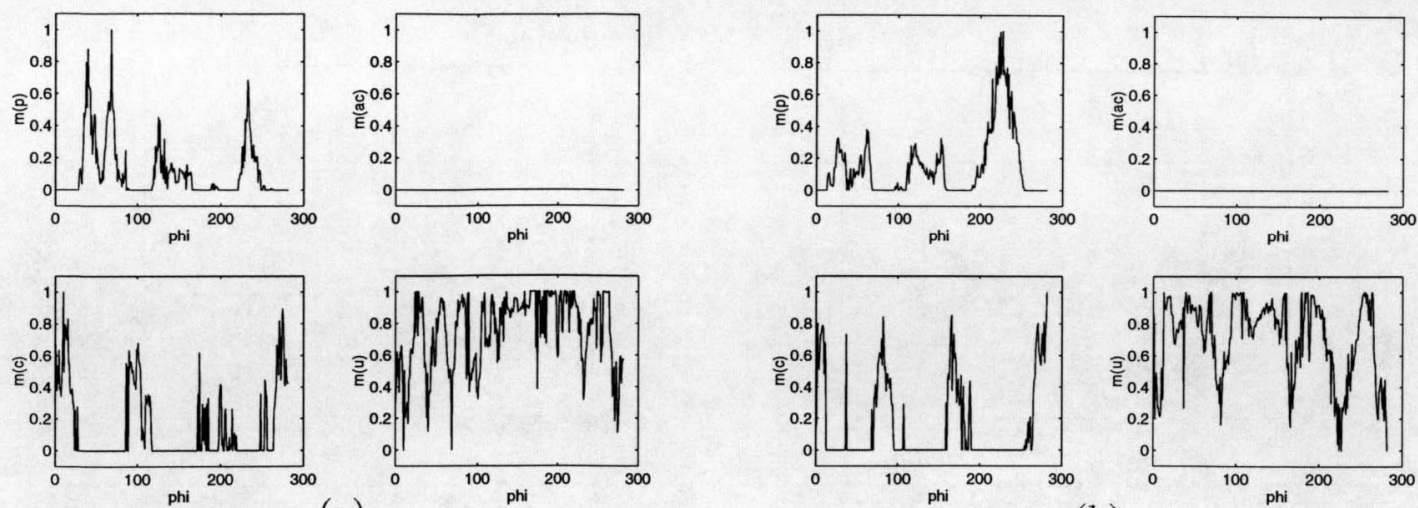

(a)
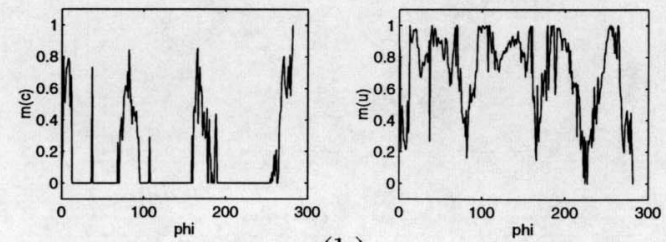

(b)
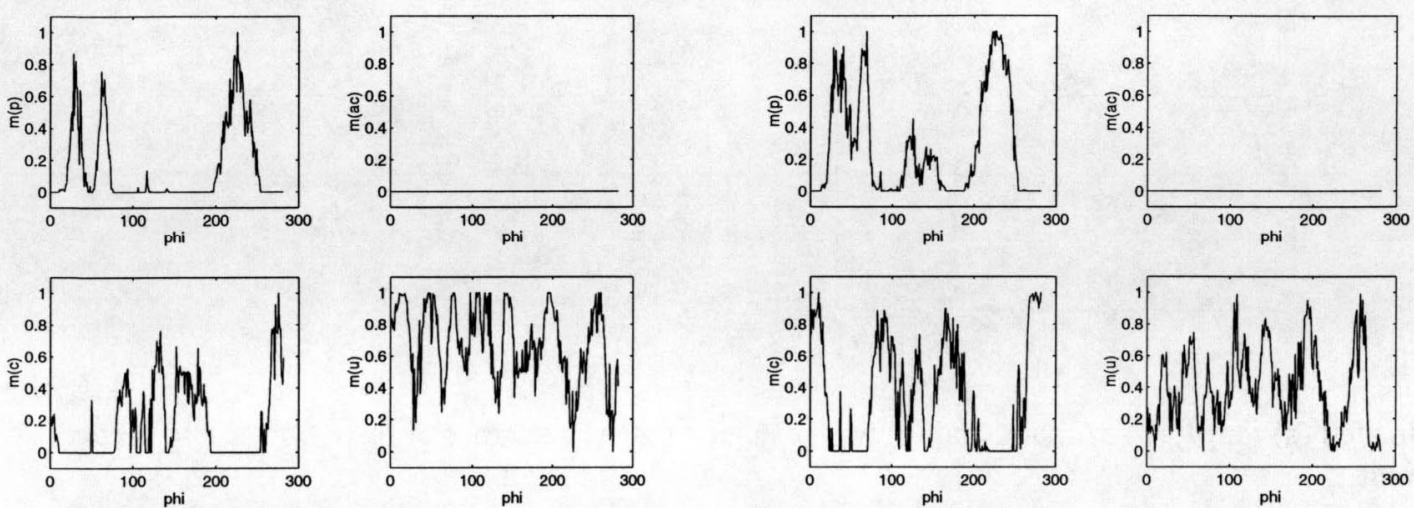

(c)
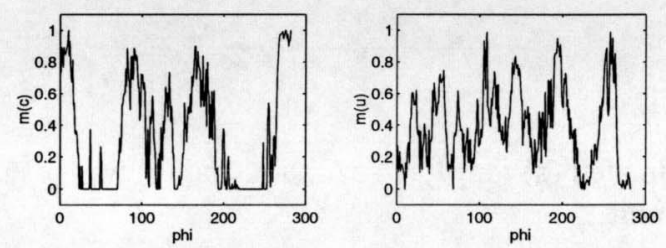

(d)

Fig. 26. Belief assignment of the sensors located at (a) $(0.0,0.0)$; (b) $(-0.1,0.1)$; (c) $(0.1,0.1)$; and (d) results when the decisions of all three sensors are fused.

From eqs. (95) and (96), one gets $\frac{r_{a}}{r_{b}}=\frac{r_{a a}}{r_{b b}} \triangleq \alpha$. Squaring and dividing eq. (97) by $r_{b}^{2}$ and using the definition of $\alpha$

$$
4 \beta^{2} \sin ^{2} \theta_{c}=\alpha^{2}+1-2 \alpha \cos \left(2 \theta_{c}-\phi_{T}\right),
$$

where $\beta \triangleq \frac{r_{a b}}{r_{b b}}$. To a good approximation, $\phi_{T} \cong 2 d / r \ll$ $2 \theta_{c}$ and can be neglected. Then, the solution for $\theta_{c}$ is

$$
\theta_{c} \cong \sin ^{-1}\left(\frac{\alpha-1}{2 \sqrt{\beta^{2}-\alpha}}\right) .
$$

Substituting for $\theta_{c}$ in eq. (95), solutions for $r_{a}$ and $r_{b}$ follow:

$$
\begin{aligned}
r_{a} & =\frac{r_{a a}}{2 \sin \theta_{c}}, \text { and } \\
r_{b} & =\frac{r_{b b}}{2 \sin \theta_{c}} .
\end{aligned}
$$

To find the locations $r$ and $\theta$ of the acute corner, the two equations

$$
\begin{aligned}
& r_{a}=\sqrt{r^{2}+\frac{d^{2}}{4}-d r \sin \theta}, \text { and } \\
& r_{b}=\sqrt{r^{2}+\frac{d^{2}}{4}+d r \sin \theta}
\end{aligned}
$$

are solved simultaneously for $r$ and $\theta$ :

$$
\begin{aligned}
& r=\sqrt{\frac{r_{a}^{2}+r_{b}^{2}}{2}-\frac{d^{2}}{4}}, \text { and } \\
& \theta=\sin ^{-1}\left(\frac{r_{b}^{2}-r_{a}^{2}}{2 d r}\right) .
\end{aligned}
$$

The TOF measurements are

$$
\begin{aligned}
t_{a a} & =\frac{r_{a a}}{c}, \\
t_{a b}=t_{b a} & =\frac{r_{a b}}{c}, \text { and } \\
t_{b b} & =\frac{r_{b b}}{c},
\end{aligned}
$$

and the signal models for each transmitter-receiver pair are given by

$$
\begin{aligned}
A_{a a}(r, \theta, d, t) & =2 \frac{A_{\max } r_{\min }}{r_{a a}} e^{-\frac{\alpha_{a a}^{2}+\beta_{a a}^{2}}{2 \sigma_{T}^{2}}} e^{-\frac{\left(t-t_{a a}-\frac{3}{f_{0}}\right)^{2}}{\sigma^{2}}} \\
& \times \sin \left[2 \pi f_{\circ}\left(t-t_{a a}\right)\right],
\end{aligned}
$$




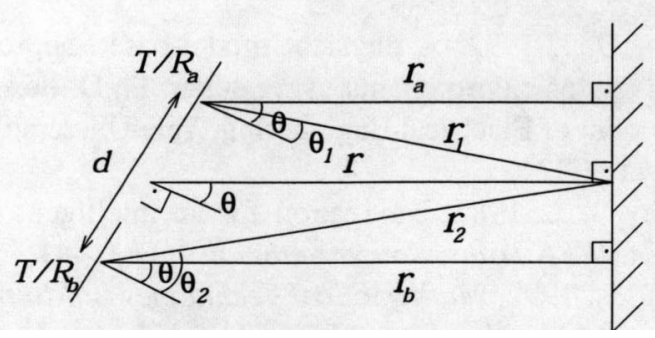

Fig. 27. Geometry of the problem with the given sensor pair when the target is a plane.

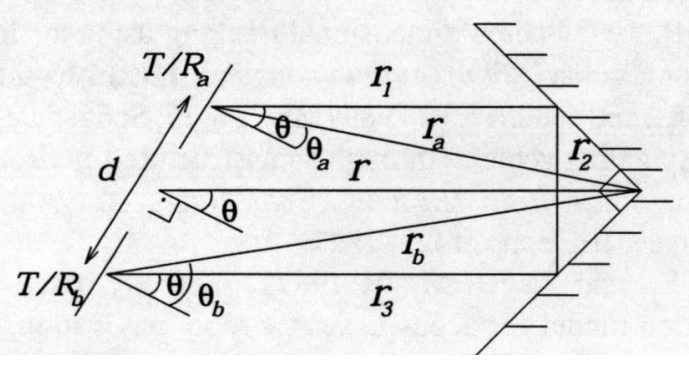

Fig. 28. Geometry of the problem when the target is a corner.

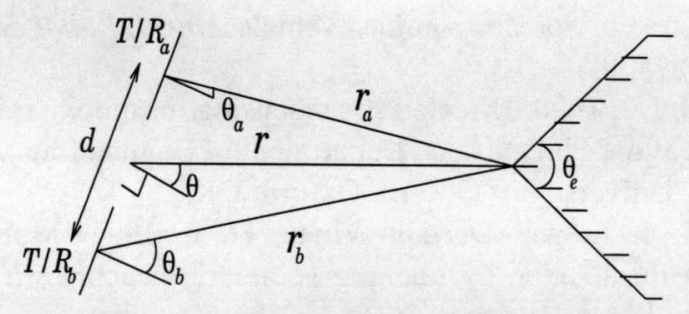

Fig. 29. Geometry of the problem when the target is an edge.

$$
\begin{aligned}
A_{a b}(r, \theta, d, t) & =2 \frac{A_{\max } r_{\min }}{r_{a b}} e^{-\frac{\alpha_{a b}^{2}+\beta_{a b}^{2}}{2 \sigma_{T}^{2}}} e^{-\frac{\left(t-t_{a b}-\frac{3}{f_{0}}\right)^{2}}{\sigma^{2}}} \\
& \times \sin \left[2 \pi f_{0}\left(t-t_{a b}\right)\right], \\
A_{b a}(r, \theta, d, t) & =2 \frac{A_{\max } r_{\min }}{r_{b a}} e^{-\frac{\alpha_{b a}^{2}+\beta_{b a}^{2}}{2 \sigma_{T}^{2}}} e^{-\frac{\left(t-t_{b a}-\frac{3}{f_{0}}\right)^{2}}{\sigma^{2}}} \\
& \times \sin \left[2 \pi f_{0}\left(t-t_{b a}\right)\right], \text { and } \\
A_{b b}(r, \theta, d, t) & =2 \frac{A_{\max } r_{\min }}{r_{b b}} e^{-\frac{\alpha_{b b}^{2}+\beta_{b b}^{2}}{2 \sigma_{T}^{2}}} e^{-\frac{\left(t-t_{b b}-\frac{3}{\sigma^{2}}\right)^{2}}{\sigma^{2}}} \\
& \times \sin \left[2 \pi f_{0}\left(t-t_{b b}\right)\right] .
\end{aligned}
$$

Here, $\alpha_{a b}$ is the angle made by the line of sight of transducer $a$ and the line drawn between the centers of transducer $a$ and virtual receiver $b$. Similarly, $\beta_{a b}$ is the angle made by the line of sight of virtual receiver $b$ and the line drawn between the centers of transducers $a$ and virtual receiver $b$. Definitions of $\alpha_{a a}, \alpha_{b b}, \beta_{a a}$, and $\beta_{b b}$ are similar.

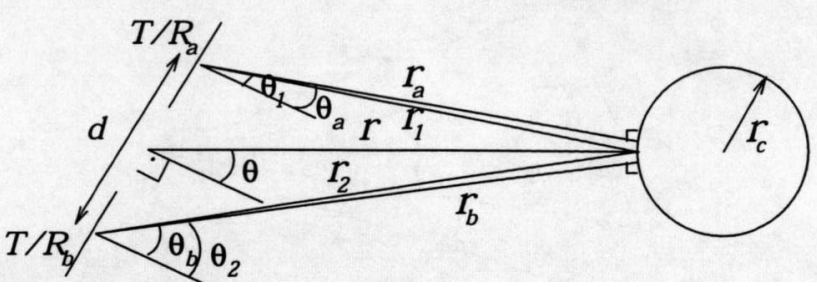

Fig. 30. Geometry of the problem when the target is a cylinder with radius $r_{c}$.
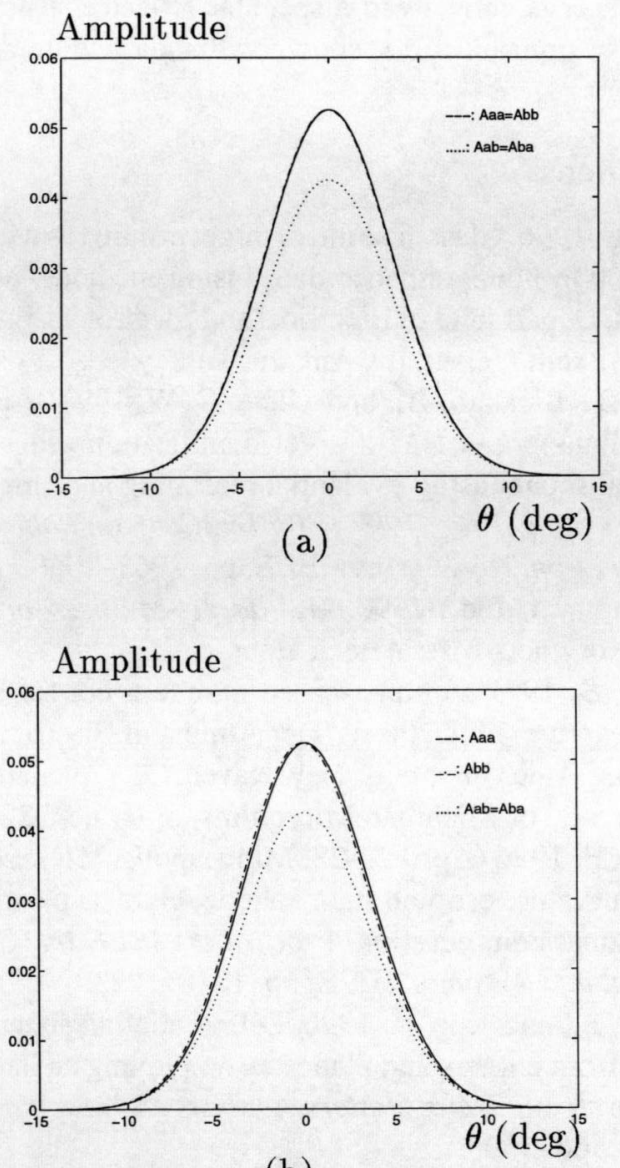

(b)

Fig. 31. Amplitude curves of the detected signal in the case of (a) a planar target, (b) and a cylindrical target with radius $10 \mathrm{~m}$. 


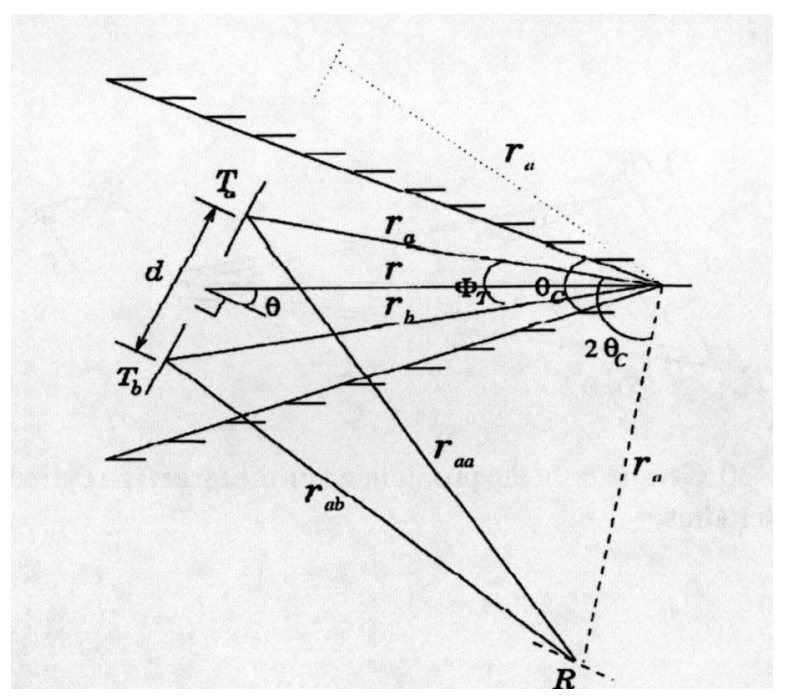

Fig. 32. Geometry of the problem where each surface of the acute corner is considered a specular reflector. The virtual receiver of transducer $a$ is shown with a dashed line.

\section{References}

Ayrulu, B. 1996. Classification of target primitives with sonar using two nonparametric data fusion methods. Master's thesis, Department of Electrical and Electronics Engineering, Bilkent University, Ankara, Turkey.

Ayrulu, B., Barshan, B., and Utete, S. W. 1997 (April 2025 , Albuquerque, NM). Target identification with multiple logical sonars using evidential reasoning and simple majority voting. Proc. 1997 IEEE Int. Conf. on Robotics and Automation. New Jersey: IEEE, pp. 2063-2068.

Bar-Shalom, Y. (ed.) 1990. Multitarget-Multisensor Tracking. Norwood, MA: Artech House.

Barshan, B. 1991. A sonar-based mobile robot for bat-like prey capture. Ph.D. thesis, Department of Electrical Engineering, Yale University, New Haven, CT. (Available from University of Michigan Microfilms, order no. 9224325.)

Barshan, B. 1996 (April 22-28, Minneapolis, MN). Location and curvature estimation of spherical targets using a flexible sonar configuration. Proc. of the IEEE Int. Conf. on Robot. and Automat. IEEE, pp. 1218-1223.

Barshan, B., and Kuc, R. 1990. Differentiating sonar reflections from corners and planes by employing an intelligent sensor. IEEE Trans. Pattern Analysis and Machine Intell. 12(6):560-569.

Berger, J. O. 1988. Statistical Decision Theory and Bayesian Analysis. Berlin: Springer-Verlag.

Blackman, S. S., and Broida, T. J. 1990. Multiple sensor data association and fusion in aerospace applications. J. Robot. Sys. 7(3):445-485.

Borenstein, J., and Koren, Y. 1988. Obstacle avoidance with ultrasonic sensors. IEEE Trans. Robot. Automat. $4(2): 213-218$.
Bozma, Ö. I. 1992. A physical model-based approach to analysis of environments using sonar. Ph.D. thesis, Department of Electrical Engineering, Yale University, New Haven, CT.

Crowley, J. L. 1985. Navigation for an intelligent mobile robot. IEEE Trans. Robot. Automat. 1(1):31-41.

Hall, D. L. 1992. Mathematical Techniques in Multisensor Data Fusion. Norwood, MA: Artech House.

Kleeman, L., and Kuc, R. 1995. Mobile robot sonar for target localization and classification. Int. J. Robot. Res. 14(4):295-318.

Kuc, R. 1993. Three-dimensional tracking using qualitative bionic sonar. Robot. Autonomous Sys. 11(2):213-219.

Kuc, R., and Barshan, B. 1989 (May 14-19, Scottsdale, AZ). Navigating vehicles through an unstructured environment with sonar. Proc. IEEE Int. Conf. Robot. Automat. New Jersey: IEEE, pp. 1422-1427.

Kuc, R., and Siegel, M. W. 1987. Physically-based simulation model for acoustic sensor robot navigation. IEEE Trans. Pattern Analysis and Machine Intell. 9(6):766778.

Kuc, R., and Viard, B. V. 1991. A physically based navigation strategy for sonar-guided vehicles. Int. J. Robot. Res. 10(2):75-87.

Leonard, J. J. 1990. Directed sonar sensing for mobile robot navigation. Ph.D. thesis, Department of Engineering Science, University of Oxford, Oxford, UK.

Leonard, J. J., and Durrant-Whyte, H. F. 1991. Mobile robot localization by tracking geometric beacons. IEEE Trans. Robot. Automat. 7(3):376-382.

Luo, R. C., and Lin, M. H. 1988 (April 24-29, Philadelphia, PA). Robot multi-sensor fusion and integration: Optimum estimation of fused sensor data. Proc. IEEE Int. Conf. on Robot. and Automat. New Jersey: IEEE, pp. 24-29.

Manyika, J., and Durrant-Whyte, H. F. 1994. Data Fusion and Sensor Management: A Decentralized InformationTheoretic Approach. New York: Ellis Horwood.

Morse, P. M., and Ingard, K. U. 1968. Theoretical Acoustics. New York: McGraw-Hill.

Murphy, R. R. 1993 (May 2-6, Atlanta, GA). Robust sensor fusion for teleoperations. Proc. IEEE Int. Conf. on Robot. and Automat. New Jersey: IEEE, pp. 572-577.

Murphy, R. R. 1996 (December 8-11, Washington, DC). Adaptive rule of combination for observations over time. Proc. IEEE/SICE/RSJ Int. Conf. on Multisensor Fusion and Integration for Intell. Sys. New Jersey: IEEE, pp. 125131.

Nazlıbilek, S., Erkmen, A., and Erkmen, I. 1993 (August, Chicago, IL). SENS-PERCEPTOR: Image-based evidence formation module as a logical sensor for robot hand preshaping. Proc. IEEE Int. Sym. on Intell. Control. New Jersey: IEEE, pp. 326-331.

Pagac, D., Nebot, E. M., and Durrant-Whyte, H. F. 1996 (April 22-28, Minneapolis, MN). An evidential ap- 
proach to probabilistic map-building. Proc. 1996 IEEE Int. Conf. on Robot. and Automat. New Jersey: IEEE, pp. 745-750.

Panasonic Corp. 1989. Ultrasonic ceramic microphones. 12 Blanchard Road, Burlington, MA.

Pearl, J. 1988. Probabilistic Reasoning in Intelligent Systems: Networks of Plausible Inference. San Mateo, CA: Morgan Kaufmann.

Peremans, H., Audenaert, K., and Van Campenhout, J. M. 1993. A high-resolution sensor based on tri-aural perception. IEEE Trans. Robot. Automat. 9(1):36-48.

Polaroid Corp. 1990. Ultrasonic components group. 119 Windsor St., Cambridge, MA.
Shafer, G. 1976. A Mathematical Theory of Evidence. Princeton, NJ: Princeton University Press.

Steinberg, A. N. 1987. (June, Naval Air Development Center, Warminster, PA). Threat management system for combat aircraft. Proc. of the Tri-Service Data Fusion Symposium, vol. 1, pp. 532-554.

Utete, S. W., Barshan, B., and Ayrulu, B. Forthcoming. Voting as validation in robot programming. Int. J. Robot. Res.

Zemanek, J. 1971. Beam behavior within the nearfield of a vibrating piston. J. Acoustical Soc. America 49(1/2):181191. 